\title{
Análisis de los niveles de conocimiento financiero usando el operador OWA:
}

\section{caso Boyacá, Colombia}

\author{
Jorge Romero-Muñoz ${ }^{1}$ \\ Universidad Pedagógica y Tecnológica de Colombia \\ jorge.romero@uptc.edu.co \\ Gina Fonseca-Cifuentes ${ }^{2}$ \\ Universidad Pedagógica y Tecnológica de Colombia \\ gina.fonseca01@uptc.edu.co \\ Fabio Blanco-Mesa ${ }^{3}$ \\ Universidad Pedagógica y Tecnológica de Colombia \\ fabio.blanco01@uptc.edu.co
}

DOI: https://doi.org/10.21158/01208160.n91.2021.3091

Fecha de recepción: 10 de julio de 2020

Fecha de aprobación: 27 de agosto de 2021

Fecha de publicación: 16 de diciembre de 2021

Cómo citar este artículo: Romero-Muñoz, J.; Fonseca-Cifuentes, G.; Blanco-Mesa, F. (2021). Análisis de los niveles de conocimiento financiero usando el operador OWA: caso Boyacá, Colombia. Revista Escuela de Administración de Negocios, (91), páginas. DOI: https://doi.org/10.21158/01208160.n91.2021.3091

\section{Resumen}

El objetivo principal de esta investigación es analizar el nivel de conocimiento financiero de los habitantes del departamento de Boyacá. Para esto se realiza un análisis descriptivo y un análisis híbrido con el operador de agregación de la media ponderada ordenada - OWA, desde tres perspectivas: el hábito de ahorro, el uso de servicios financieros y la mejora de la educación financiera. La investigación considera tres conceptos fundamentales sobre conocimientos financieros básicos, conformados por el interés compuesto, la inflación y la diversificación del riesgo. Así mismo, se considera la metodología de la Superintendencia Financiera de Colombia, con el fin de usar conceptos propios del entorno que permitan la contextualización del participante frente a interrogantes del mercado financiero nacional. Para esto se determina la muestra de estudio de forma probabilística por provincias, que corresponde a un total de 1914 unidades y se presentan las cinco preguntas que permiten brindar una aproximación fiable sobre conocimientos básicos en finanzas. Los hallazgos muestran que los boyacenses presentan dificultad en comprender las capacidades de diversificar y diferenciar el riesgo, lo cual afecta el nivel de conciencia y de responsabilidad de sus decisiones financieras. Como conclusión se destaca que la debilidad en el conocimiento financiero restringe el desarrollo de hábitos financieros que permitan otorgar mayor empoderamiento a las personas, por lo cual se hace pertinente la ejecución de planes de acción que redunden en alianzas público-privadas, de acuerdo con la Estrategia Nacional de Educación Económica y Financiera de Colombia.

Palabras clave: conocimiento financiero; evaluación de conocimiento financiero; educación financiera; alfabetización financiera; hábito de ahorro; uso de servicios financieros.

\footnotetext{
${ }^{1}$ Magister en Administración de Empresas - Universidad Externado de Colombia. Especialista en Finanzas - Universidad Pedagógica y Tecnológica de Colombia. Administrador de Empresas - Universidad Pedagógica y Tecnológica de Colombia. ORCID: https://orcid.org/0000-0003-4952-2435

2 Magister en Administración Económica y Financiera - Universidad Tecnológica de Pereira. Especialista en Finanzas - Universidad Pedagógica y Tecnológica de Colombia. Administradora de Empresas -Universidad Pedagógica y Tecnológica de Colombia. ORCID: https://orcid.org/0000-0003$1497-4802$

${ }^{3}$ Ph. D. Internacional en Empresa - Universidad de Barcelona. Máster en Investigación en Empresa, Finanzas y Seguros y Máster en Dirección de Empresas del Deporte - Universidad de Barcelona. Administrador Deportivo de la Universidad Distrital Francisco José de Caldas. ORCID: https://orcid.org/0000-0002-9462-6498
} 


\title{
Analysis of Financial Literacy Levels Using the OWA Operator: the Case of Boyacá, Colombia
}

\begin{abstract}
The primary objective of this research is to analyze the level of financial knowledge of residents in the Department of Boyacá. For this purpose, we carried out descriptive and hybrid analyses using the ordered weighted average (OWA) aggregation operator from three perspectives: the habit of saving, the use of financial services, and the improvement of financial education. The research considers three fundamental concepts of basic financial literacy: compound interest, inflation, and risk diversification. In the same way, we considered the methodology of the Superintendencia Financiera de Colombia [Financial Superintendence of Colombia] to use concepts in this environment that allow for contextualization in the face of questions on the national financial market. For this purpose, the study sample is determined probabilistically by provinces, corresponding to 1914 units; and the five questions that allow providing a reliable approximation of basic financial knowledge are presented. The findings show that people from the Department of Boyacá have difficulty understanding the ability to diversify and differentiate risk, which affects the level of awareness and responsibility when making financial decisions. In conclusion, we highlight that the weakness in financial knowledge restricts the development of financial habits that allow granting greater empowerment to people. Thus, it becomes pertinent to implement action plans that result in public-private alliances in accordance with the National Strategy for the Economic and Financial Education in Colombia.
\end{abstract}

Keywords: financial knowledge; financial knowledge assessment; financial education; financial literacy; habit of saving; use of financial services.

Análise dos níveis de conhecimento financeiro usando a operadora OWA: caso Boyacá, Colômbia

\section{Resumo}

O objetivo principal desta pesquisa é analisar o nível de conhecimento financeiro dos moradores da região de Boyacá. Para isso, foram realizadas uma análise descritiva e uma análise híbrida com o operador de agregação da média ponderada ordenada - OWA, a partir de três perspectivas: o hábito de economizar, a utilização de serviços financeiros e a melhoria da educação financeira. A pesquisa considera três conceitos fundamentais sobre o conhecimento financeiro básico, formados por juros compostos, pela inflação e pela diversificação de riscos. Da mesma forma, é considerada a metodologia da Superintendência Financeira da Colômbia, a fim de utilizar conceitos do seu próprio meio que permitam a contextualização do participante diante das questões do mercado financeiro nacional. Para isso, a amostra do estudo é determinada probabilisticamente por províncias, o que corresponde a um total de 1.914 unidades e são apresentadas as cinco questões que permitem oferecer uma aproximação fiável dos conhecimentos básicos em finanças. Os resultados mostram que as pessoas de Boyacá têm dificuldade em compreender as capacidades de diversificar e diferenciar o risco, do qual afeta 0 nível de consciência e de responsabilidade de suas decisões financeiras. Como conclusão, destaca-se que a debilidade do conhecimento financeiro restringe o desenvolvimento de hábitos financeiros que permitam conceder maior poder às pessoas, por isso é pertinente a execução de planos de ação que resultem em alianças público-privadas, de acordo com a Estratégia Nacional de Educação Econômica e Financeira da Colômbia.

Palavras-chave: conhecimento financeiro; avaliação de conhecimento financeiro; educação financeira; alfabetização financeira; hábito de economizar; uso de serviços financeiros. 
Analyse des connaissances financières grâce à l'opérateur OWA: cas du département de Boyacá, Colombie

\begin{abstract}
Résumé
L'objectif principal de cette recherche est l'analyse des connaissances financières des habitants du département de Boyacá. Une analyse descriptive et une hybride seront réalisées avec l'opérateur d'agrégation de moyenne pondérée ordonnée OWA, selon trois perspectives : la culture de l'épargne, l'utilisation des services financiers et l'amélioration de l'éducation financière. Ce travail prend en compte trois concepts fondamentaux sur les connaissances financières de base et l'intérêt composé, l'inflation et la diversification des risques. La méthodologie de l'opérateur de surveillance du système financier colombien sera prise en compte afin d'utiliser des concepts permettant une contextualisation des participants aux thématiques du marché financier national. Cette étude porte sur un échantillon déterminé de manière probabiliste par province, et correspond à un total de 1914 unités. Les cinq questions soumises aux participants permettent une approximation fiable des connaissances financières de base. Les résultats montrent que les habitants de Boyacá éprouvent des difficultés à comprendre les possibilités de diversification et de différenciation des risques, affectant ainsi le niveau de conscience et de responsabilité de leurs décisions financières. En conclusion, le manque de connaissances financières limite le développement d'habitudes financières saines qui permettraient aux participants de jouir d'une plus grande autonomie ; c'est pourquoi la mise en place de programmes d'action au travers d'alliances public-privé, conformément à la loi, pourrait être une stratégie nationale d'éducation économique et financière pertinente.
\end{abstract}

Mots-clés: connaissances financières; évaluation des connaissances financières; éducation financière; littérature financière; habitude d'épargne; recours aux services financiers.

\title{
1. Introducción
}

En su contexto social y cultural, las personas se encuentran constantemente relacionadas con términos y actividades financieras que determinan en el mediano y largo plazo su bienestar económico. Bajo esta realidad surge la necesidad de establecer estrategias que permitan mejorar los conocimientos financieros. En este sentido, las acciones que se desarrollan a través de la educación pueden ser útiles para mejorar la comprensión de los diferentes términos financieros. Así, el desarrollo de habilidades financieras por medio de la acción de alfabetización y educación financiera ha adquirido varias acepciones en las que se pueden identificar tres variables básicas relacionadas con el conocimiento en conceptos financieros, la capacidad de comprender estos conceptos y las habilidades para tomar decisiones financieras.

El primero es fundamental en el desarrollo de una adecuada educación financiera (Kim, Kratzer y Leech, 2001), ya que mejora la alfabetización financiera y puede tener efectos positivos en la toma de decisiones, así como desarrollar mejores competencias financieras en 
las personas. La segunda destaca la relevancia que tiene la capacidad de comprender los conceptos financieros (Lusardi y Tufano, 2009), esencial en el procesamiento de la información financiera y en el momento de tomar decisiones sobre su planificación, el endeudamiento, las inversiones y las pensiones (Lusardi y Mitchell, 2014). La tercera se destaca por el desarrollo de las habilidades para tomar decisiones financieras que sean llevadas a la práctica (Zait y Bertea, 2014). También es importante destacar que la cultura tiene influencia en los niveles de conocimiento financiero en los que la incertidumbre y el individualismo tienen influencias significativas para su desarrollo (De Beckker, De Witte y Van Campenhout, 2020).

Estas aproximaciones sugieren que las acciones de alfabetización y educación financiera tienen un rol importante en la formación social en un entorno financiero, en el cual el conocimiento y las habilidades financieras fortalezcan las competencias financieras básicas que deben tener las personas. En este sentido, los programas de alfabetización financiera pueden tener efectos positivos en el conocimiento y el comportamiento financiero de las personas (Kaiser et al., 2020). Además, una adecuada formación financiera puede ofrecer las herramientas para afrontar crisis financieras como la recientes (Cordero y Pedraja, 2018). Por tanto, las acciones encaminadas a la alfabetización financiera tienen mayor importancia en las decisiones económicas cotidianas (Goyal y Kumar, 2021), en las que es indispensable el fomento de estrategias nacionales que mejoren los niveles de conocimiento financiero.

Dado que es importante conocer cuál es el nivel de los conocimientos financieros de las personas, estos son susceptibles de ser medidos. La evaluación de esos conocimientos permite un diagnóstico de los niveles de educación financiera que tienen las personas en relación con su entorno. Por esta razón, su evaluación y medición son fundamentales en el propósito de comprender y autoevaluar la percepción sobre conocimientos y habilidades en este campo. En este sentido, se han desarrollado y presentado diversas propuestas que van desde pruebas representadas en porcentajes (Mandell, 2008; Volpe, Kotel y Chen, 2002) hasta pruebas múltiples y dicotómicas con diferentes cantidades de ítems a evaluar (Lusardi 
y Mitchell, 2006; 2007; 2008). Por tanto, estas pruebas intentan mostrar la capacidad y el conocimiento de las competencias financieras de las personas.

En Colombia estos niveles de conocimiento financiero que afectan la educación financiera demuestran analfabetismo generalizado en alusión a conceptos básicos referidos con la inflación, las tasas de interés y la comprensión de la relación riesgo/rentabilidad asociada al mercado de capitales. Se presentan dificultades en la interpretación de la expresión «tasa de interés», de manera que se dificulta la comprensión de cálculos básicos sobre tasa de interés simple o compuesta en más de la mitad de la población.

Así mismo, el concepto inflación muestra las mismas dificultades, pues son incomprendidos cálculos afines con los cambios del poder adquisitivo del dinero y con el entendimiento de la relación riesgo y diversificación del portafolio. Sin embargo, este escenario se ve disminuido en población con mayores ingresos - mayores ingresos en la población, mayores conocimientos financieros- (García et al., 2013). También se destaca que en las mujeres el indicador de conocimiento financiero es bajo y se hace más relevante frente a una mayor cantidad de hijos y al no tener acceso a este tipo de conocimiento (Vanegas et al., 2020).

Por tanto, la precariedad sobre conocimientos financieros en los colombianos impide el acceso y el uso consciente a los productos y servicios ofertados, aun en un mayor grado cuando estas definiciones mantienen un carácter técnico (Corporación Andina de Fomento, 2015). Igualmente, sobre las conductas relacionadas con diferentes situaciones financieras, la toma de decisiones, en promedio, no es la adecuada, agudizada en la medida en que se tiene un menor nivel educativo, menor estrato socioeconómico o que se es residente de zonas rurales (CAF et al., 2015).

Estas razones precisaron la construcción del Conpes 4005 de 2020 «Política Nacional de Inclusión y Educación Económica y Financiera», amparado, principalmente, en la Ley 1328 de 2009 —-derecho a la educación financiera-y el Decreto 457 de 2014 —Sistema Administrativo Nacional para la Educación Económica y Financiera-, cuyo objetivo principal consiste en «integrar los servicios financieros a las actividades cotidianas de los 
ciudadanos y las mipymes para contribuir al crecimiento e inclusión financiera del país» (Consejo Nacional de Política Económica y Social, 2020, p. 58), y que define a la educación económica y financiera como,

[...] el proceso a través del cual los individuos desarrollan los valores, conocimientos, competencias y comportamientos necesarios para la toma de decisiones financieras responsables, que requieren la aplicación de conceptos financieros básicos y el entendimiento de los efectos que los cambios en los principales indicadores macroeconómicos generan en su nivel de bienestar. (Como se cita en Departamento Nacional de Planeación [DNP], 2011)

Finalmente, en Colombia, la totalidad de programas de educación o alfabetización financiera, en su mayoría de instituciones financieras, carecen de componentes de evaluación en su impacto. Se encontró que, de cien programas, tan solo tres tienen una evaluación rigurosa y sus efectos son a corto plazo (Álvarez-Franco et al., 2017), lo cual evidencia la necesidad de realizar investigaciones sobre el nivel de conocimiento financiero de las personas.

Con base en lo anterior, el objetivo principal de esta investigación es analizar el nivel de conocimiento financiero de los habitantes del departamento de Boyacá, con lo cual se busca evaluar los conocimientos financieros y comparar los resultados teniendo en cuenta las variables de hábito de ahorro, uso de servicios financieros y adecuada educación financiera. Para esto se utilizan como referentes los estudios de Lusardi y Mitchell (2014) y de la Superintendencia Financiera de Colombia y se lleva a cabo un análisis descriptivo y un análisis híbrido con el operador de agregación OWA, propuesto por Blanco-Mesa, GilLafuente y Merigó (2018b), desde tres perspectivas: el hábito de ahorro, el uso de servicios financieros y la mejora de la educación financiera.

Los hallazgos muestran bajos niveles de conocimientos básicos en finanzas en los boyacenses, de modo que se destacan dificultades en la comprensión para diversificar y diferenciar el riesgo, lo que puede afectar el nivel de conciencia y de responsabilidad de la toma de decisiones financieras. En conclusión, el bajo nivel en conocimientos financieros limita el desarrollo de los hábitos financieros que permitan otorgar mayor empoderamiento 
de las personas y mejorar los niveles de educación financiera. El presente documento se estructura de la siguiente manera: en la sección 2 se presentan las aproximaciones teóricas sobre educación, evaluación y medición financiera. En la sección 3 se encuentra la metodología que contempla el caso de estudio, el análisis descriptivo y el análisis híbrido. En la sección 4 se presentan los principales resultados de la investigación. Finalmente, en la sección 5 se encuentran las principales conclusiones e implicaciones de la investigación.

\section{Marco teórico}

En esta sección se presentan las aproximaciones sobre el concepto de educación financiera y las diversas formas de evaluación que se han utilizado para medir el nivel de educación financiera, los cuales orientan el desarrollo de la investigación.

\subsection{Una aproximación al conocimiento financiero}

La educación financiera ha tenido diversas interpretaciones, lo cual ha llevado a establecer una discusión sobre los factores que debe incluir una buena educación financiera. El concepto de educación financiera puede contemplar tres variables: el conocimiento en conceptos financieros, la capacidad de comprender estos conceptos y las habilidades para tomar decisiones financieras. Así, sobre estas aproximaciones se puede dar a entender qué es la educación financiera y por qué tiene un rol importante en la formación social en un entorno financiero.

El conocimiento de los conceptos financieros es fundamental en el desarrollo de una adecuada educación financiera (Braunstein y Welch, 2002; Hilgert y Hogarth, 2003; Kim et al., 2001; Lusardi y Mitchell, 2007; Moore, 2003; Remund, 2010). Por tanto, la comprensión de aspectos financieros es prioritaria para la sobrevivencia en nuestra sociedad (Kim et al., 2001), puesto que puede mejorar el bienestar financiero de las personas (Braunstein y Welch, 2002). Así, la alfabetización financiera depende de un buen conocimiento financiero para ser más competentes (Hilgert y Hogarth, 2003). 
Según Moore (2003), las personas son más competentes cuando usan los conocimientos financieros que han aprendido, lo que genera sofisticación en la toma de decisiones. Así mismo, Lusardi y Mitchell (2007) afirman que es necesario tener conocimientos básicos en conceptos financieros a fin de tomar decisiones razonables en ahorro e inversión, así como son ineludibles para la toma de decisiones financieras a corto y largo plazo (Remund, 2010). Por tanto, una adecuada alfabetización financiera puede tener efectos positivos en la toma de decisiones y formar personas con mejores competencias financieras.

El conocimiento financiero es importante, no obstante, es relevante la capacidad que se debe tener para comprender sus conceptos (Chen y Volpe, 1998; Emmons, 2005; Faulcon-Bowen, 2002; Fox, Bartholomae y Lee, 2005; Lusardi y Tufano, 2009; Lusardi y Mitchell, 2014; Schagen y Lines, 1996). Esta capacidad está relacionada con la gestión, el análisis y la comprensión de las circunstancias financieras que afectan las personas (Chen y Volpe, 1998). Así mismo, el desarrollo de la capacidad de entendimiento de estos conceptos es clave para el funcionamiento en la sociedad (Faulcon-Bowen, 2002), lo que demuestra un mejor manejo del ahorro, el financiamiento, el efectivo, los seguros de vida y los planes para necesidades financieras por parte de las personas (Emmons, 2005).

De acuerdo con Fox et al. (2005), la educación financiera es la capacidad decisiva en la toma decisiones efectivas para el consumidor, lo que permite hacer juicios informados con respecto al uso del dinero (Schagen y Lines, 1996). De igual manera, Lusardi y Tufano (2009) afirman que es la capacidad de simplificar las decisiones sobre conceptos financieros en contextos de cotidianidad. Así, se puede considerar que el desarrollo de esta capacidad por parte de las personas ayudaría en el procesamiento de la información financiera y a tomar decisiones sobre su planificación, el endeudamiento, las inversiones y las pensiones (Lusardi y Mitchell, 2014).

Con conocimiento y comprensión, las habilidades para tomar decisiones financieras deben mejorar (Kozup y Hogarth, 2008; Olin Gutiérrez, 2014; Stone, Wier y Bryant, 2008; Toussaint-Comeau y Rhine, 2000; Zait y Bertea, 2014). Estas habilidades sobre conocimientos financieros son necesarias para tomar decisiones financieras apropiadas, las 
cuales se ven reflejadas en los valores personales de las personas (Stone et al., 2008; Toussaint-Comeau y Rhine, 2000). Así mismo, estas habilidades son de pensamiento crítico para apreciar y valorar las decisiones financieras en relación con las necesidades y objetivos que tengan las personas (Kozup y Hogarth, 2008). Así, en el proceso de toma de decisiones la comprensión de los conceptos financieros se ven reflejados con el desarrollo de habilidades llevadas a la práctica (Olin-Gutiérrez, 2014; Zait y Bertea, 2014).

En este sentido, la importancia del conocimiento, el entendimiento y la efectividad busca tener un consenso y entendimiento general a partir de la educación. Según la Organización para la Cooperación y el Desarrollo Económicos (OCDE) (2005),

la educación financiera es el proceso por el cual los consumidores/inversores financieros mejoran su conocimiento sobre los productos, conceptos y riesgos financieros y, a través de información, instrucción y/o consejo objetivo, desarrollan las habilidades y confianza para adquirir una mayor concienciación de los riesgos y oportunidades financieras, para tomar decisiones informadas, para saber dónde acudir para pedir ayuda y adoptar otras medidas efectivas para mejorar su bienestar financiero.

Así, desde procesos formales se puede lograr la intensión de promover, desarrollar y mejorar los conocimientos y las habilidades financieras de las personas.

Basado en lo anterior, se observa que las aproximaciones hechas sobre el conocimiento financiero y su intencionalidad de tener personas mejor educadas y conscientes son amplias y contemplan diversos aspectos que la hacen compleja. En este sentido, se presenta un acercamiento conceptual sencillo que permite su fácil uso y recordación. Esta aproximación tiene en cuenta los componentes semánticos de las tres variables descritas y se formula así: «el conocimiento financiero se entiende como el grado de conocimiento de los conceptos financieros, la capacidad y habilidad de entenderlos, para el uso efectivo en la toma de decisiones a corto y largo plazo de su vida». 
En conclusión, estas variables se interrelacionan una y otra vez en la vida cotidiana de las personas, ya sea en una negociación empresarial o en un acuerdo familiar sobre los gastos del hogar. Esto ha conllevado a que el concepto de educación financiera tenga dificultades en su tratamiento, debido a su generalizada importancia en la sociedad y en las personas, dado que su buena o inadecuada utilización acarrea cambios significativos en la orientación financiera de estas.

\subsection{Evaluación y medición del conocimiento financiero}

Es habitual que, a fin de medir el grado de conocimiento y la comprensión de los conceptos financieros se usen diversas formas de evaluación. Se han propuesto varias aproximaciones sobre los métodos utilizados para evaluar y medir los conocimientos financieros, los cuales terminan enfocándose en medir el valor del dinero en el tiempo, el mercado de capitales, las tasas de interés, el endeudamiento, el sistema financiero, la inflación, el ahorro, el crédito, la diversificación del portafolio, los flujos de efectivo de los hogares y la jubilación, entre otros, pero sin comprender la capacidad de entendimiento de las personas sobre estos (Lusardi y Mitchell, 2014).

Así mismo, se han propuesto otras metodologías, como, por ejemplo, las pruebas de rendimiento sobre conceptos financieros y las autoevaluaciones que permiten valorar la percepción sobre conocimientos y habilidades financieras. Por ejemplo, Chen y Volpe (1998) y Volpe et al. (2002) proponen una prueba de porcentaje con diez ítems y 36 ítems con opción múltiple, y Mandell (2008) elabora una prueba de conocimiento con porcentaje. Además, Hilgert y Hogarth (2003) utilizan una prueba de conocimiento con porcentajes, mientras Moore (2003) desarrolla un informe sobre las experiencias financieras con catorce ítems. Igualmente, Mandell (2006) lleva a cabo una prueba de conocimiento con 31 ítems y Agnew y Szykman (2005) presentan una prueba de diez ítems con opción múltiple y de verdadero y falso.

De manera similar y más amplia, Lusardi y Mitchell (2006; 2007; 2008) desarrollan una prueba con tres elementos con opción múltiple y de falso y verdadero, luego una prueba de 
los tres elementos computacionales y, finalmente, una prueba de cinco ítems básicos de respuestas de opción múltiple y ocho ítems sofisticados de opción múltiple. En otra propuesta, Van Rooij, Lusardi y Alessie (2011) plantean dos pruebas de cinco ítems y once ítems de educación financiera básica y sofisticada con opción múltiple. De modo similar, Lusardi y Tufano (2009) confeccionan una prueba de tres ítems con opción múltiple. Como se ha descrito, se han desarrollado diferentes propuestas que evalúan la capacidad y el conocimiento de las competencias financieras de las personas.

Por su parte, la Superintendencia Financiera de Colombia (2018), como parte del Banco de la República y la Comisión Intersectorial para la Educación, Económica y Financiera (CIEEF, 2017), a partir de la identificación de actividades transversales que propendan a la inclusión de iniciativas para el fortalecimiento de estrategias de educación financiera que permitan el desarrollo de metodologías y contenidos, realiza como recurso didáctico la metodología Superfinanzas a través de un juego de mesa.

Este juego, como herramienta lúdica, contiene definiciones, características y factores relevantes para la comprensión de los mercados financieros en el país, cuyo propósito es contestar de forma correcta el mayor número de preguntas, lo que permite el afianzamiento de conocimientos sobre productos y servicios del sector financiero, de tal manera que inserta al participante dentro de una dinámica que genera la discusión, el debate y la ampliación de conocimientos por medio de otras fuentes.

Teniendo en cuenta la revisión de las diferentes metodologías, la presente investigación considera el trabajo desarrollado por Lusardi y Mitchell (2014), el cual se orienta por tres conceptos fundamentales sobre conocimientos financieros básicos, conformados por el interés compuesto, la inflación y la diversificación del riesgo (véase la Tabla 1). Así mismo, se considera la metodología de la Superintendencia Financiera de Colombia, con el fin de usar conceptos propios del entorno que permitan la contextualización del participante frente a interrogantes del mercado financiero nacional, de modo que exista una adecuada interpretación de la pregunta y, por ende, no haya lugar a ambigüedad en la respuesta. 
Así pues, la utilización de estas metodologías se hace porque contempla aspectos esenciales que debe tener una prueba de conocimiento, de entendimiento y de forma oral, como son el nivel de simplicidad, la relevancia, la brevedad y la capacidad de diferenciar las preguntas (Lusardi y Mitchell, 2014).

Tabla 1. Preguntas base sobre educación financiera

\begin{tabular}{|l|l|l|}
\hline \multicolumn{1}{|c|}{$\mathbf{P}$} & \multicolumn{1}{|c|}{ Enunciado } & \multicolumn{1}{c|}{ Respuestas } \\
\hline 1 & $\begin{array}{l}\text { Imagine que tiene cien dólares en una cuenta de ahorros } \\
\text { que le renta un tipo de interés anual del 2\%. Al cabo de } \\
\text { cinco años, ¿qué saldo cree que tendría en la cuenta si } \\
\text { mantuviese el dinero depositado generando intereses? }\end{array}$ & $\begin{array}{l}\text { • Más de USD 102 } \\
\text { Exactamente USD 102 } \\
\text { • Menos de USD 102 } \\
\text { • No sabe/no contesta }\end{array}$ \\
\hline 2 & $\begin{array}{l}\text { Supongamos que el tipo de interés de su cuenta de } \\
\text { ahorros es del 1 \% al año y que la tasa de inflación anual } \\
\text { es del 2 \%. Al cabo de un año, ¿qué cantidad de bienes } \\
\text { cree que podría comprar con el dinero de dicha cuenta? }\end{array}$ & $\begin{array}{l}\text { • Más que hoy } \\
\text { Exactamente la misma } \\
\text { que hoy } \\
\text { - Menos que hoy } \\
\text { - No sabe/no contesta }\end{array}$ \\
\hline 3 & $\begin{array}{l}\text { Califique la siguiente afirmación como verdadera o falsa: } \\
\text { invertir en acciones de una única empresa suele generar } \\
\text { una rentabilidad más segura que invertir en } \\
\text { participaciones de un fondo de inversión. }\end{array}$ & \\
\hline
\end{tabular}

Fuente. Lusardi y Mitchell, 2014.

La primera pregunta se refiere a los conocimientos sobre las tasas de interés compuesto por medio de la capacidad matemática - aritmética básica- de los encuestados. La segunda pregunta observa el conocimiento que tienen los encuestados en economía básica, como lo es la inflación. La tercera pregunta observa sí las personas encuestadas tienen conocimiento sobre la diversificación del riesgo en las inversiones. Estas preguntas se han consolidado en todo el mundo como instrumento de medición sobre la educación financiera básica.

\section{Metodología}

El presente trabajo de investigación pretende mostrar los niveles de educación financiera de Boyacá; se realizó en el 2018 en las diferentes provincias de este departamento. Para esto se determina la muestra de estudio de forma probabilística por provincias, que corresponde a un total de 1914 unidades, se presentan las cinco preguntas que permiten brindar una aproximación fiable sobre conocimientos básicos en finanzas, las cuales fueron extractadas 
de los estudios de Lusardi y Mitchell (2014) y la Superintendencia Financiera de Colombia. A fin de realizar el tratamiento de los datos obtenidos se lleva a cabo un análisis descriptivo y un análisis híbrido con el operador de agregación OWA propuesto por Blanco-Mesa et al. (2018b). Estos análisis permitirán mostrar el nivel de conocimientos financieros de los boyacenses y cómo el nivel educativo se relaciona con el hábito de ahorro, el uso de servicios financieros y la mejora de la educación financiera.

\subsection{Provincias del departamento de Boyacá}

En el departamento de Boyacá se tiene una población aproximada de 1255311 habitantes (DANE, 2007), distribuidos en un total de trece provincias y dos zonas de manejo especial Puerto Boyacá y el distrito fronterizo de Cubará-. Estas provincias son agrupadas en cinco regiones proporcionales en cantidad de población. La muestra obtenida es de 1914 con un nivel de confianza del 95 \% y un margen de error de 5 \%, la ratio de respuesta es del $100 \%$ (véase la Tabla 2). Como criterio de selección de los participantes en estas provincias se tiene en cuenta la oferta de servicios financieros en los municipios, ya que con mayor oferta de servicios financieros es más probable desarrollar capacidades para hacer efectivo el uso del sistema.

Tabla 2. Municipios de las provincias en Boyacá - Población 2005

\begin{tabular}{|c|c|c|c|}
\hline Provincia Sugamuxi - $\mathrm{Su}-$ & Municipios que la conforman & Población & Muestra \\
\hline Sugamuxi & $\begin{array}{l}\text { Aquitania, Cuítiva, Firavitoba, Gámeza, Iza, Mongua, Monguí, } \\
\text { Nobsa, Pesca, Sogamoso, Tibasosa, Tópaga, Tota }\end{array}$ & 201569 & \multirow{4}{*}{383} \\
\hline Valderrama & Chita, Paz del Río, Socha, Socotá, Tasco, Betéitiva y Jericó & 46322 & \\
\hline Libertad & Labranzagrande, Pajarito, Pisba & 11476 & \\
\hline \multicolumn{2}{|r|}{ 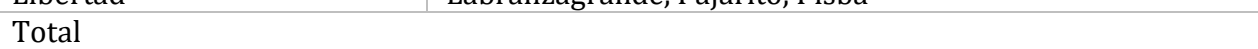 } & 259367 & \\
\hline Provincia Gutiérrez — Gu- & Municipios que la conforman & Población & Muestra \\
\hline Gutiérrez & $\begin{array}{l}\text { Chiscas, Cubará, El Cocuy, El Espino, Guacamayas, Güicán, } \\
\text { Panqueba }\end{array}$ & 30677 & \multirow{4}{*}{383} \\
\hline Tundama & $\begin{array}{l}\text { Belén, Busbanzá, Cerinza, Corrales, Duitama, Floresta, Paipa, } \\
\text { Santa Rosa de Viterbo, Tutazá }\end{array}$ & 166546 & \\
\hline Norte & $\begin{array}{l}\text { Boavita, Covarachía, La Uvita, San Mateo, Sativanorte, } \\
\text { Sativasur, Soatá, Susacón, Tipacoque }\end{array}$ & 37578 & \\
\hline \multicolumn{2}{|l|}{ Total } & 234801 & \\
\hline
\end{tabular}




\begin{tabular}{|c|c|c|c|}
\hline Provincia Centro - $\mathrm{Ce}-$ & Municipios que la conforman & Población & Muestra \\
\hline Centro & $\begin{array}{l}\text { Chíquiza, Chivata, Cómbita, Cucaita, Motavita, Oicatá, Samacá, } \\
\text { Siachoque, Sora, Soracá, Sotaquirá, Toca, Tunja, Tuta, } \\
\text { Ventaquemada }\end{array}$ & 262978 & \multirow{3}{*}{383} \\
\hline Márquez & $\begin{array}{l}\text { Boyacá, Ciénaga, Jenesano, Nuevo Colón, Ramiriquí, Rondón, } \\
\text { Tibaná, Turmequé, Úmbita, Viracachá }\end{array}$ & 66005 & \\
\hline \multicolumn{2}{|r|}{ 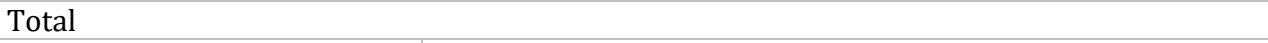 } & 329033 & \\
\hline Provincia Lengupá —Le- & Municipios que la conforman & Población & Muestra \\
\hline Lengupá & $\begin{array}{l}\text { Berbeo, Campohermoso, Miraflores, Páez, San Eduardo, } \\
\text { Zetaquirá }\end{array}$ & 25391 & \multirow{4}{*}{382} \\
\hline Neira & $\begin{array}{l}\text { Chinavita, Garagoa, Macanal, Pachavita, San Luis de Gaceno, } \\
\text { Santa María }\end{array}$ & 38081 & \\
\hline Oriente & $\begin{array}{l}\text { Almeida, Chivor, Guateque, Guayatá, La Capilla, Somondoco, } \\
\text { Sutatenza, Tenza }\end{array}$ & 35491 & \\
\hline \multicolumn{2}{|l|}{ Total } & 98963 & \\
\hline $\begin{array}{l}\text { Provincia Occidente y } \\
\text { Ricaurte }-\mathrm{Oc}-\end{array}$ & Municipios que la conforman & Población & Muestra \\
\hline Occidente & $\begin{array}{l}\text { Briceño, Buenavista, Caldas, Chiquinquirá, Coper, La Victoria, } \\
\text { Maripí, Muzo, Otanche, Pauna, Puerto Boyacá, Quípama, } \\
\text { Saboyá, San Miguel de Sema, San Pablo de Borbur, Tununguá }\end{array}$ & 197512 & \multirow{3}{*}{383} \\
\hline Ricaurte & $\begin{array}{l}\text { Arcabuco, Chitaraque, Gachantivá, Moniquirá, Ráquira, } \\
\text { Sáchica, San José de Pare, Santa Sofía, Santana, Sutamarchán, } \\
\text { Tinjacá, Togüí, Villa de Leyva }\end{array}$ & 91569 & \\
\hline \multicolumn{2}{|r|}{ 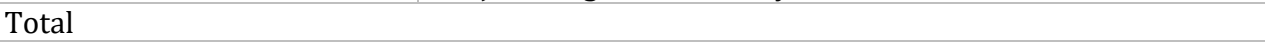 } & 289081 & \\
\hline \multicolumn{2}{|l|}{ Muestra Total para el estudio } & & 1914 \\
\hline
\end{tabular}

Nota. No se tiene en cuenta las poblaciones de las zonas de manejo especial.

Fuente. Elaboración propia con base a datos obtenidos del DANE, 2007.

\subsection{Determinación de las preguntas sobre conocimiento financiero}

Para la determinación de las preguntas que permitan conocer el nivel de conocimiento financiero de los boyacenses se tiene en cuenta la propuesta teórica y metodológica de Lusardi y Mitchell (2014), así como de la Banca de las Oportunidades y la Superintendencia Financiera de Colombia (2018). La propuesta de Lusardi y Mitchell (2014) se fundamenta en tres conceptos principales: la capacidad de realizar cálculos relacionados con los tipos de interés y la comprensión de la inflación y de la diversificación del riesgo. Estos conceptos son operacionalizados en tres preguntas - P1 a P3 - caracterizadas por la simplicidad, la relevancia, la brevedad y la capacidad de diferenciar (Lusardi y Mitchell 2014).

Por otro lado, la propuesta de la Banca de las Oportunidades y la Superintendencia Financiera de Colombia (2018) se fundamenta en conceptos relacionados con productos y servicios de establecimientos de crédito. Estos conceptos son operacionalizados en dos preguntas - $\mathrm{P} 4 \mathrm{y}$ 
P5-, caracterizadas desde la inclusión y la utilización de productos financieros, lo que permitiría justificar, en términos generales, mayor discernimiento frente a estos temas. Así, tomando los conceptos originales se adaptan con el fin de proponer cinco preguntas con múltiples alternativas y una única respuesta correcta. Es de notar cómo la fundamentación teórica que define estas preguntas viene dada de los estudios mencionados. Así mismo, las preguntas hacen parte de la configuración central de la construcción del instrumento utilizado. Por tanto, en la tabla 3 se muestra la adaptación de las preguntas para el contexto de estudio y las posibles soluciones a partir de los conceptos definidos.

Tabla 3. Preguntas sobre conocimiento financiero

\begin{tabular}{|c|c|c|c|}
\hline Concepto & $\mathbf{N}$ & Pregunta & PS \\
\hline Tipos de interés & $\mathrm{P}_{1}$ & $\begin{array}{l}\text { Suponga que tiene } \$ 1000000 \text { en una cuenta } \\
\text { de ahorros y que el banco le da el } 2 \% \text { anual a } \\
\text { su cuenta. ¿Cuánto dinero tiene en la cuenta } \\
\text { después de cinco años si nunca retiró nada? }\end{array}$ & $\begin{array}{l}\text { - Más de } \$ 1100000 \\
\text { - Exactamente } \$ 1100000 \\
\text { - Menos de } \$ 1100000 \\
\text { - Ninguna de las anteriores }\end{array}$ \\
\hline $\begin{array}{l}\text { Comprensión de la } \\
\text { inflación }\end{array}$ & $\mathrm{P}_{2}$ & $\begin{array}{l}\text { Supongamos que usted tiene } \$ 1000000 \text { para } \\
\text { comprar bienes y la inflación se mantiene en } \\
2 \% \text { anual. ¿Luego de un año, usted que } \\
\text { supondría? }\end{array}$ & $\begin{array}{l}\text { - Compraría menos de lo que puede } \\
\text { comprar hoy en día } \\
\text { - Compraría la misma cantidad de lo } \\
\text { que puede comprar hoy en día } \\
\text { - Compraría más de lo que puede } \\
\text { comprar hoy en día } \\
\text { - Ninguna de las anteriores }\end{array}$ \\
\hline $\begin{array}{l}\text { Diversificación del } \\
\text { riesgo }\end{array}$ & $\mathrm{P}_{3}$ & $\begin{array}{l}\text { Supongamos que usted tiene una suma de } \\
\text { dinero. Consideraría más seguro poner su } \\
\text { dinero en: }\end{array}$ & $\begin{array}{l}\text { - Un negocio o inversión } \\
\text { - Múltiples negocios o inversiones } \\
\text { - Ninguna de las anteriores }\end{array}$ \\
\hline $\begin{array}{l}\text { Servicios } \\
\text { Tasa de interés }\end{array}$ & $\mathrm{P}_{4}$ & $\begin{array}{l}\text { ¿Cuál es la tasa interés real que cobra el } \\
\text { sistema financiero? }\end{array}$ & $\begin{array}{l}\text { - Tasa nomina } \\
\text { - Tasa mensual vencido } \\
\text { - Tasa efectiva anual } \\
\text { - Ninguna de las anteriores }\end{array}$ \\
\hline Producto financiero & $\mathrm{P}_{5}$ & Firmar un cheque en la parte de atrás es... & $\begin{array}{l}\text { - Liberarlo } \\
\text { - Endosarlo } \\
\text { - Validarlo } \\
\text { - Ninguna de las anteriores }\end{array}$ \\
\hline
\end{tabular}

Nota. PS: posibles soluciones; N: número de pregunta.

Fuente. Elaboración propia con base en el trabajo de Lusardi y Mitchell, 2014 y Superfinanciera, 2018. 


\subsection{Operador OWA y su extensión fuzzy significance (FS)}

La media ponderada ordenada — su acrónimo en inglés OWA operator (Yager, 1988) - es un tipo de media ponderada en la que los argumentos se asocian a un vector de peso para obtener un único valor representativo con el fin de establecer un orden ascendente o descendente según el criterio establecido por el decisor. El criterio del decisor está asociado con los parámetros de optimismo/pesimismo, los cuales se ubican dentro de un valor mínimo y un valor máximo (Baez-Palencia, Olazabal-Lugo, y Romero-Muñoz, 2019; Blanco-Mesa, Gil-Lafuente y Merigo, 2018a; Blanco-Mesa, León-Castro y Merigó, 2019; Blanco-Mesa, León-Castro y Acosta-Sandoval, 2020; Blanco-Mesa, León-Castro y GilLafuente, 2019). El operador se define de la siguiente manera:

Definición 1. Un operador OWA de la dimensión n es un mapeo $O W A: R^{n} \rightarrow R$ que tiene asociado un vector de ponderación $\mathrm{W}$ de la dimensión $\mathrm{n}$ con $w_{j} \in[0,1] \mathrm{y} \sum_{j=1}^{n} w_{j}=1$, tal que:

$$
O W A\left(a_{1}, a_{2}, \ldots, a_{n}\right)=\sum_{j=1}^{n} w_{j} b_{j}
$$

Donde $b_{\mathrm{j}}$ es el jth el más grande de los $a_{\mathrm{i}}$.

El operador fuzzy significance OWAD (FS-OWAD) propuesto por Blanco-Mesa et al. (2018b) agrega la información según el significado de sus características. Esta permite tener en cuenta a un cierto nivel todas las características, es decir, se considera la importancia de la relación de cada característica para tomar una decisión. Para tratar un problema de toma de decisiones, se realiza la siguiente propuesta.

Definición 2. Un operador OWAD con fuzzy significance puede tener un vector de peso $W$, $\sum_{j=1}^{n} \frac{w_{j}}{\max \left(w_{j}\right)}, \mathrm{y} w_{j} \in[0,1]$ tal que:

$$
F S-O W A D\left(\left\langle x_{1}, y_{1}\right\rangle, \ldots,\left\langle x_{n}, y_{n}\right\rangle\right)=\sum_{j=1}^{n} \frac{w_{j}}{\max \left(w_{j}\right)} D_{j},(2)
$$

Donde $D_{j}$ representa el $j t h$ el más grande de los $\left|x_{i}-y_{i}\right|$. 
Este operador no cumple con la condición de límite e idempotencia, ya que es fácil obtener un resultado superior al máximo. Esto sucede porque la suma de los pesos puede ser superior a uno (Blanco-Mesa et al., 2018c). A fin de cumplir con la condición de frontera se puede utilizar un proceso de normalización en el que la suma de los pesos se convierte en uno (Gil-Lafuente y Merigó, 2009). Un método práctico para hacerlo es dividir los pesos por la suma de todos ellos. Por tanto, este operador es muy útil como método para la toma de decisiones, pero no representa la información desde un punto de vista clásico.

\section{Resultados}

En esta sección se presentan los resultados obtenidos del tratamiento descriptivo y el híbrido. En el primer tratamiento los resultados obtenidos se enfocan en los conocimientos básicos de educación financiera en relación con las regiones, el género, la edad y el nivel educativo. En el segundo tratamiento, los resultados obtenidos se enfocan en los conocimientos básicos de educación financiera con un nivel ideal de respuesta y como variables mediadoras el hábito de ahorro, el uso de servicios financieros y la mejora de la educación financiera. A continuación, se presentan los resultados obtenidos de ambos tratamientos.

\subsection{Análisis descriptivo}

En el desarrollo del análisis descriptivo se tomó la población del departamento de Boyacá, Colombia, la cual es de 1255311 habitantes, aproximadamente; se obtiene una muestra de 1914 personas encuestadas mayores de edad, dividida en cinco grupos de regiones, las cuales se caracterizan por las variantes que enlistan a continuación.

- Género. De los encuestados, el $51 \%$ son mujeres y el $49 \%$ son hombres.

- Rangos de edades. Entre 18-29 años un $27 \%$, entre 30-39 años un $23 \%$, entre 40-49 años un $24 \%$ y mayores de 50 a más años un $26 \%$ de los encuestados. 
- Estrato socioeconómico. El estrato 1 con un $24 \%$, el estrato 2 con un 34 \%, el estrato 3 con un $36 \%$, el estrato 4 con un $5 \%$ y el estrato 5 con un $1 \%$ de los encuestados.

- Nivel educativo. Ninguno $4 \%$, primaria $5 \%$, bachillerato $35 \%$, técnico $18 \%$, pregrado 24 \%, especialización 9 \%, maestría 2 \% y otros 3 \%.

Con esta caracterización inicial se procede a hacer el análisis de conocimientos básicos de educación financiera de las personas encuestadas. En la tabla 4 se observa el porcentaje de aciertos que se obtuvo al dar respuesta a las cinco preguntas. Se evidencia que la pregunta P1 es la que menor ratio de acierto tiene y que la pregunta P5 es la que mayor ratio de acierto tiene. Estos resultados sugieren, por una parte, que las personas tienen dificultad en comprender la capacidad de diversificar y diferenciar el riesgo, y, por otra, las personas comprenden la simplicidad de un instrumento bancario.

De acuerdo con Bruhn et al. (2013), y García-Bohórquez (2012), el crecimiento y la complejidad de los servicios y los productos financieros conlleva a la limitada y restringida información sobre estos, aunando a la desacertada toma de decisiones, prácticas financieras inapropiadas e incapacidad de defensa sobre los derechos como consumidores financieros.

Por otro lado, según la Banca de las Oportunidades y la Superintendencia Financiera de Colombia (2018), en el país cerca de 26,8 millones de personas son poseedores de al menos un producto de depósito, lo cual demuestra avances significativos en materia de inclusión financiera, por lo que el acceso a productos financieros ha significado en Boyacá que el porcentaje de adultos con uso frecuente de estos productos alcance el 82,2 \%, lo cual denota que, si bien es significativo el avance frente a la inclusión financiera, existen brechas, entre otros factores, sobre la comprensión de la capacidad adquisitiva y la gestión del dinero.

Así mismo, se destaca el bajo ratio de acierto de las preguntas restantes, lo que ratifica también la dificultad que tienen las personas en hacer cálculos sobre el valor del dinero y comprender conceptos esenciales del sistema financiero. Estas dificultades pueden restringir el entendimiento del acceso al sistema financiero al no saber cómo usarlo y no comprender los servicios que estas entidades ofrecen, lo cual puede conllevar a que las personas tengan 
una débil planeación de sus finanzas personales. De esta manera, es pertinente que la educación financiera propicie autoconciencia sobre el riesgo e inductores hacia la conducta del ahorro que estimulen el aumento de la capacidad en la toma de decisiones financieras y coadyuve al incremento de la confianza y las habilidades en la determinación de objetivos financieros, así como a la fijación de medios pertinentes para su cumplimiento (Lewis y Messy, 2012).

Tabla 4. Conocimientos básicos de educación financiera

\begin{tabular}{|l|c|c|c|c|c|}
\hline & $\mathrm{P} 1$ & $\mathrm{P} 2$ & $\mathrm{P} 3$ & $\mathrm{P}$ & $\mathrm{P} 5$ \\
\hline Correcto & $16,3 \%$ & $43,4 \%$ & $37,4 \%$ & $39,1 \%$ & $53,5 \%$ \\
\hline Incorrecto & $83,7 \%$ & $56,6 \%$ & $62,6 \%$ & $60,9 \%$ & $46,5 \%$ \\
\hline
\end{tabular}

Fuente. Elaboración propia.

Basado en los análisis anteriores, se muestra cómo se distribuyen estos resultados de acuerdo con las regiones y el nivel educativo. En la figura 1 se presentan las provincias, y se observa que la provincia de Sugamuxi — Su - tiene las ratios de respuesta más bajos en las preguntas P1, P2, P3 y P4, mientras la provincia Centro - Ce - tiene la ratio de respuesta más bajo en la pregunta P5. En cuanto a las ratios más altas se distribuyen de la siguiente manera: para P1, P3, P4 y P5 la provincia de Occidente - Oc-, y para P2 la provincia de Gutiérrez - Gu一. 
Figura 1. Conocimientos básicos de educación financiera/regiones
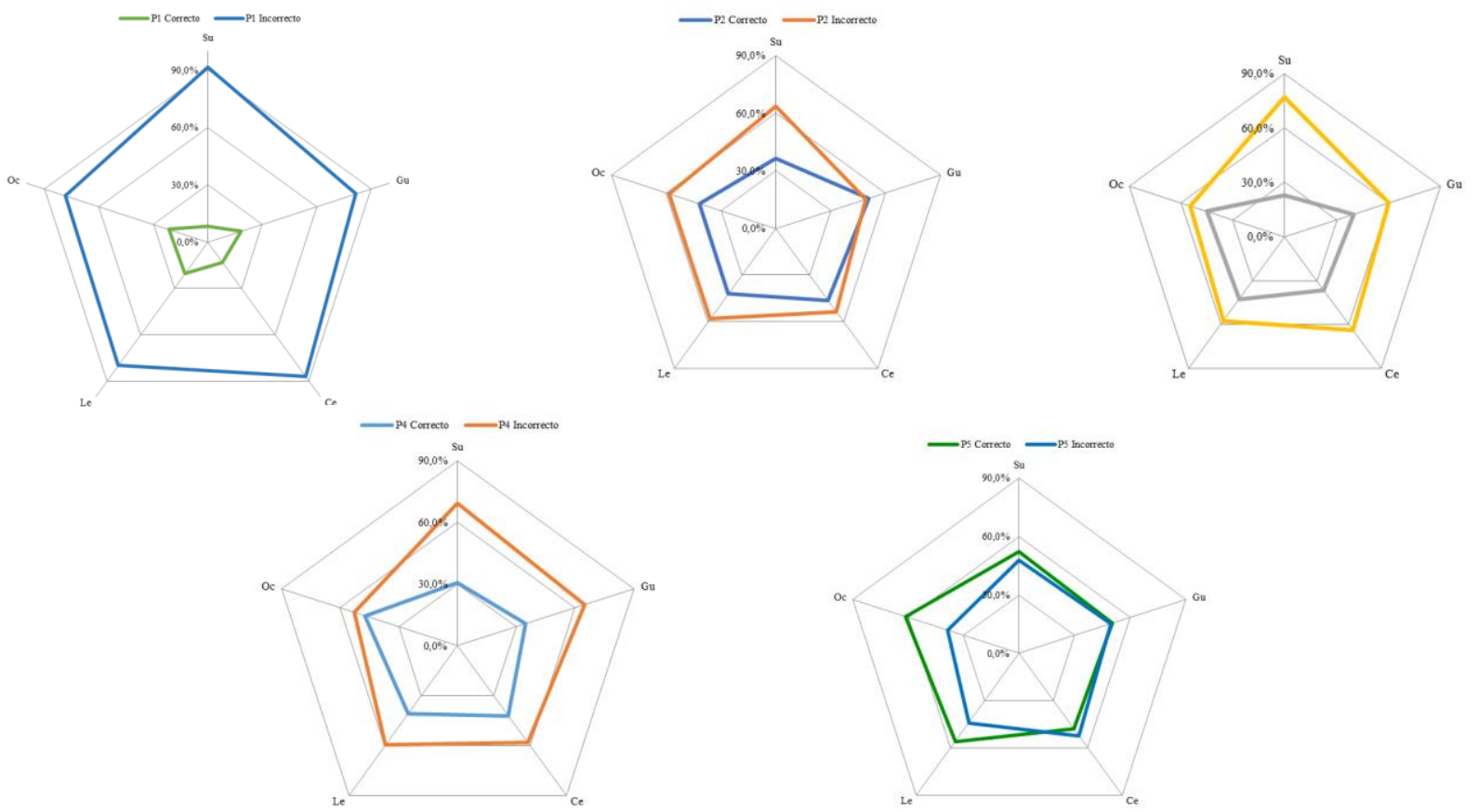

Fuente. Elaboración propia. 
Figura 2. Conocimientos básicos de educación financiera/nivel educativo
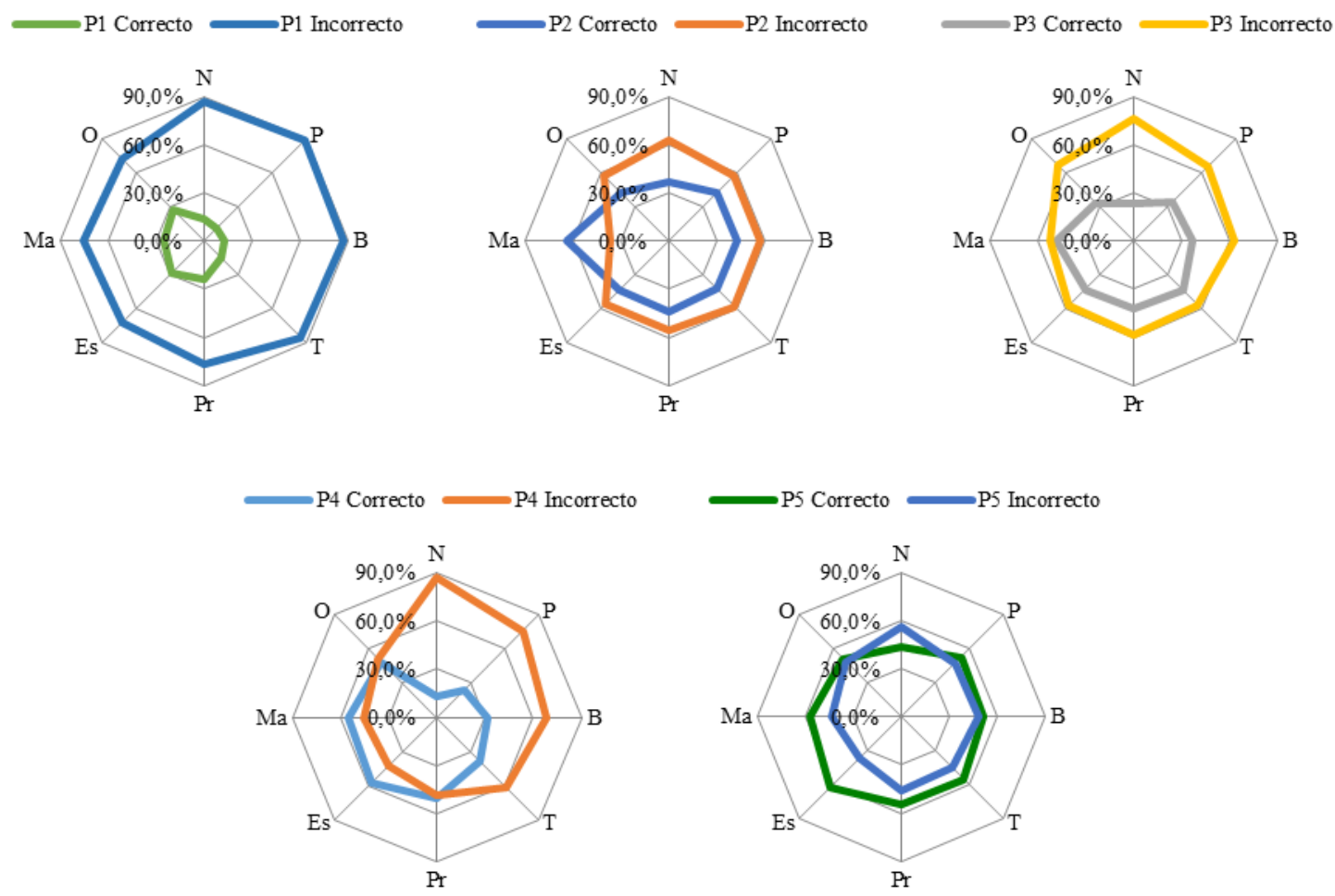

Nota. N: ninguno; P: primaria; B: bachillerato; T: técnico; Pr: pregrado; Es: especialización; Ma: maestría.

Fuente. Elaboración propia.

Cabe aclarar que los resultados obtenidos son negativos dado que la ratio de acierto es inferior a la ratio de desaciertos, exceptuando la pregunta P5. En la figura 2 se muestra el nivel educativo, en el que se observa una variación importante de los resultados; esta es evidente al destacar que a mayor nivel educativo más alta es la ratio de acierto de las respuestas. Así, por ejemplo, en todas las preguntas, excepto P1, la ratio de acierto es superior a la ratio de desaciertos, de modo que se destacan las personas que tienen educación posgraduada, como, por ejemplo, especialización y maestría. Un aspecto que llama la atención es la diferencia mínima que existe entre las personas con estudios técnicos y de pregrado. 


\section{Análisis híbrido}

En la literatura ya se han realizado análisis con metodologías que permitan desarrollarlos mediante técnicas alternativas para el análisis el conocimiento financiero como, por ejemplo, el machine learning (Levantesi et al., 2021). En este estudio se usa el operador OWA como herramienta híbrida, el cual se caracteriza por agregar y comparar la información según con el significado de sus características, es decir, la importancia que tiene la información más que su propia medición.

Con este análisis se busca determinar qué tanto pueden mejorar las precisiones de las respuestas teniendo en cuenta los aciertos según el nivel educativo y las tres variables de peso, como lo son el hábito de ahorro, el uso de servicios financieros y la adecuada educación financiera. En la tabla 5 se muestra el ideal que debe existir en el acierto de las respuestas, es decir, el valor 1 significa que se deben tener un acierto del $100 \%$ en todas las respuestas. Este será el valor con el que se compara los aciertos del nivel educativo (véase la Tabla 6).

Tabla 5. Respuesta ideal

\begin{tabular}{|c|c|c|c|c|c|c|c|c|}
\hline Ideal & $\mathrm{N}$ & $\mathrm{P}$ & $\mathrm{B}$ & $\mathrm{T}$ & $\mathrm{Pr}$ & $\mathrm{Es}$ & $\mathrm{Ma}$ & $\mathrm{O}$ \\
\hline Aciertos & 1 & 1 & 1 & 1 & 1 & 1 & 1 & 1 \\
\hline
\end{tabular}

Nota. N: ninguno; P: primaria; B: bachillerato; T: técnico; Pr: pregrado; Es: especialización; Ma: maestría; O: otro.

Fuente. Elaboración propia.

Tabla 6. Aciertos nivel educativo

\begin{tabular}{|c|c|c|c|c|c|c|c|c|}
\hline & $\mathrm{N}$ & $\mathrm{P}$ & $\mathrm{B}$ & $\mathrm{T}$ & $\mathrm{Pr}$ & $\mathrm{Es}$ & $\mathrm{Ma}$ & $\mathrm{O}$ \\
\hline $\mathrm{P}_{1}$ & 0,13 & 0,11 & 0,13 & 0,15 & 0,23 & 0,28 & 0,25 & 0,27 \\
\hline $\mathrm{P}_{2}$ & 0,37 & 0,43 & 0,43 & 0,42 & 0,44 & 0,44 & 0,64 & 0,42 \\
\hline $\mathrm{P}_{3}$ & 0,23 & 0,34 & 0,37 & 0,44 & 0,42 & 0,43 & 0,48 & 0,33 \\
\hline $\mathrm{P}_{4}$ & 0,13 & 0,24 & 0,32 & 0,39 & 0,51 & 0,57 & 0,55 & 0,48 \\
\hline $\mathrm{P}_{5}$ & 0,43 & 0,53 & 0,52 & 0,55 & 0,54 & 0,63 & 0,57 & 0,52 \\
\hline
\end{tabular}

Nota. N: ninguno; P: primaria; B: bachillerato; T: técnico; Pr: pregrado; Es: especialización; Ma: maestría; O: otro.

Fuente. Elaboración propia. 
Como variables mediadoras y de carácter importante se usan los tres vectores de peso para cada uno de los niveles educativos, el vector de pesos W se encuentra utilizando el carácter actitudinal es tal que $\alpha=\square(1 /(r+1))$, y si especificamos $\alpha$ podemos obtener $r=\square((1-\alpha) / \alpha)$ (Yager, 2009) (véanse las tablas 7, 8 y 9), los cuales obtienen con base en la opinión —criterio del individuo- que tienen las personas sus hábitos de ahorro, uso de servicios financieros y mejora en la educación financiera de acuerdo con sus niveles educativos.

Tabla 7. Vectores de peso: hábito de ahorro

\begin{tabular}{|l|c|c|c|c|c|c|c|c|}
\hline & $W_{\mathrm{N}}$ & $W_{\mathrm{P}}$ & $W_{\mathrm{B}}$ & $W_{\mathrm{T}}$ & $W_{\text {Pr }}$ & $W_{\text {Es }}$ & $W_{\text {Ma }}$ & $W_{\mathrm{O}}$ \\
\hline $\mathrm{HAH}$ & 20,0 & 70,0 & 70,3 & 77,1 & 77,4 & 86,5 & 90,9 & 75,8 \\
\hline $\mathrm{N}$ & 0,22 & 0,77 & 0,77 & 0,85 & 0,85 & 0,95 & 1,00 & 0,83 \\
\hline
\end{tabular}

Nota. HAH: hábito de ahorro; N: normalización.

Fuente. Elaboración propia.

Tabla 8. Vectores de peso: uso de servicios financieros

\begin{tabular}{|l|c|c|c|c|c|c|c|c|}
\hline & $W_{\mathrm{N}}$ & $W_{\mathrm{P}}$ & $W_{\mathrm{B}}$ & $W_{\mathrm{T}}$ & $W_{\text {Pr }}$ & $W_{\text {Es }}$ & $W_{\text {Ma }}$ & $W_{0}$ \\
\hline USF & 40,0 & 53,1 & 54,7 & 66,5 & 73,8 & 94,4 & 88,6 & 75,8 \\
\hline $\mathrm{N}$ & 0,42 & 0,56 & 0,58 & 0,70 & 0,78 & 1,00 & 0,94 & 0,80 \\
\hline
\end{tabular}

Nota. USF: uso de servicios financieros; N: normalización.

Fuente. Elaboración propia.

Tabla 9. Vectores de peso: adecuada educación financiera

\begin{tabular}{|l|c|c|c|c|c|c|c|c|}
\hline & $W_{\mathrm{N}}$ & $W_{\mathrm{P}}$ & $W_{\mathrm{B}}$ & $W_{\mathrm{T}}$ & $W_{\text {Pr }}$ & $W_{\text {Es }}$ & $W_{\text {Ma }}$ & $W_{\mathrm{O}}$ \\
\hline $\mathrm{AEF}$ & 50,0 & 80,1 & 84,8 & 89,6 & 95,0 & 94,4 & 93,2 & 90,9 \\
\hline $\mathrm{N}$ & 0,53 & 0,84 & 0,89 & 0,94 & 1,00 & 0,99 & 0,98 & 0,96 \\
\hline
\end{tabular}

Nota. AEF: adecuada educación financiera; N: normalización.

Fuente. Elaboración propia. 
Después de realizar los cálculos se obtienen los resultados de comparar el ideal de las respuestas, el nivel educativo y las tres variables mediadoras. Cabe recordar que los valores correctos son los que se acercan más al límite inferior, es decir, están más cerca de 0 (véanse las tablas 10,11 y 12 ).

Tabla 10. Nivel educativo/hábito de ahorro - HAH

\begin{tabular}{|c|c|c|c|c|c|c|c|c|c|}
\hline & $\mathrm{N}$ & $\mathrm{P}$ & $\mathrm{B}$ & $\mathrm{T}$ & $\mathrm{Pr}$ & $\mathrm{Es}$ & $\mathrm{Ma}$ & $\mathrm{O}$ & Promedio \\
\hline $\mathrm{P}_{1}$ & 0,19 & 0,68 & 0,67 & 0,72 & 0,65 & 0,68 & 0,75 & 0,61 & 0,62 \\
\hline $\mathrm{P}_{2}$ & 0,14 & 0,44 & 0,44 & 0,49 & 0,47 & 0,53 & 0,36 & 0,48 & 0,42 \\
\hline $\mathrm{P}_{3}$ & 0,17 & 0,51 & 0,49 & 0,48 & 0,49 & 0,55 & 0,52 & 0,56 & 0,47 \\
\hline $\mathrm{P}_{4}$ & 0,19 & 0,59 & 0,53 & 0,52 & 0,42 & 0,41 & 0,45 & 0,43 & 0,44 \\
\hline $\mathrm{P}_{5}$ & 0,19 & 0,68 & 0,67 & 0,72 & 0,65 & 0,68 & 0,75 & 0,61 & 0,62 \\
\hline
\end{tabular}

Fuente. Elaboración propia.

Tabla 11. Nivel educativo/uso de servicios financieros - USF

\begin{tabular}{|c|c|c|c|c|c|c|c|c|c|}
\hline & $\mathrm{N}$ & $\mathrm{P}$ & $\mathrm{B}$ & $\mathrm{T}$ & $\mathrm{Pr}$ & $\mathrm{Es}$ & $\mathrm{Ma}$ & $\mathrm{O}$ & Promedio \\
\hline $\mathrm{P}_{1}$ & 0,46 & 0,75 & 0,78 & 0,80 & 0,77 & 0,71 & 0,74 & 0,70 & 0,71 \\
\hline $\mathrm{P}_{2}$ & 0,33 & 0,48 & 0,51 & 0,55 & 0,56 & 0,56 & 0,36 & 0,55 & 0,49 \\
\hline $\mathrm{P}_{3}$ & 0,40 & 0,55 & 0,56 & 0,53 & 0,58 & 0,57 & 0,51 & 0,64 & 0,54 \\
\hline $\mathrm{P}_{4}$ & 0,46 & 0,64 & 0,61 & 0,58 & 0,49 & 0,42 & 0,45 & 0,49 & 0,52 \\
\hline $\mathrm{P}_{5}$ & 0,30 & 0,40 & 0,43 & 0,42 & 0,46 & 0,37 & 0,42 & 0,46 & 0,41 \\
\hline
\end{tabular}

Fuente. Elaboración propia.

Tabla 12. Nivel educativo/adecuada educación financiera - AEF

\begin{tabular}{|c|c|c|c|c|c|c|c|c|c|}
\hline & $\mathrm{N}$ & $\mathrm{P}$ & $\mathrm{B}$ & $\mathrm{T}$ & $\mathrm{Pr}$ & Es & Ma & 0 & Promedio \\
\hline $\mathrm{P}_{1}$ & 0,37 & 0,50 & 0,50 & 0,60 & 0,60 & 0,72 & 0,70 & 0,58 & 0,57 \\
\hline $\mathrm{P}_{2}$ & 0,27 & 0,32 & 0,33 & 0,41 & 0,44 & 0,56 & 0,34 & 0,46 & 0,39 \\
\hline $\mathrm{P}_{3}$ & 0,32 & 0,37 & 0,37 & 0,40 & 0,45 & 0,57 & 0,49 & 0,54 & 0,44 \\
\hline $\mathrm{P}_{4}$ & 0,37 & 0,43 & 0,40 & 0,43 & 0,38 & 0,43 & 0,43 & 0,41 & 0,41 \\
\hline $\mathrm{P}_{5}$ & 0,24 & 0,27 & 0,28 & 0,32 & 0,36 & 0,37 & 0,41 & 0,39 & 0,33 \\
\hline
\end{tabular}

Fuente. Elaboración propia. 
Para comprender los resultados obtenidos se hace un promedio de todos los resultados con el fin de mostrar gráficamente cuál es la variable mediadora que mejoraría más la ratio de acierto de las respuestas. En la figura 3 se observa cada uno de los promedios obtenidos para cada uno de los tres análisis hechos. Dado que los resultados de los promedios se acercan a 0 , se hace el proceso de inverso aditivo con el fin de observar los resultados en valores máximos, es decir que Prom $_{\max }=1-$ Prom $_{\min }$. Al ver las tres curvas de resultados se observa que la mejora en la ratio de las respuestas se puede dar con AEF y HAH. Las mejoras se darían en más de un 50 \%, excepto en la pregunta P1. En relación con los resultados con AEF ratifican que, si se mejora la educación financiera, los conocimientos y los hábitos en las finanzas personales tendrían un mejor desempeño.

En relación con HAH es importante destacar que el hábito de ahorro es una acción recurrente que permite guardar una porción de los ingresos, el cual es inculcado inicialmente en los hogares con el uso de la alcancía. Así, la mejora en la educación financiera se puede dar si las personas comprenden la importancia del ahorro y se asocia de manera positiva con factores tales como el valor del dinero, la diversificación y la diferenciación del riesgo.

En cuanto a la mejora que se puede obtener por USF no es tan significativa y su diferencia está entre los 12 y los 6 puntos. Estos resultados permiten inferir que usar los servicios financieros no garantiza que las personas tengan una adecuada educación financiera y que este uso se hace más por la inserción que tienen las entidades financieras en el cotidiano que por la comprensión que puedan tener sobre el uso de los productos financieros que estas ofrecen.

Esto resulta contradictorio, ya que el uso de servicios financieros no garantiza una mejora en los conocimientos básicos financieros de las personas, lo que no contribuye al desarrollo de competencias ni a la responsabilidad financiera. Finalmente, los resultados obtenidos son negativos en cuanto a los conocimientos básicos en finanzas que tienen los boyacenses, lo cual afecta el nivel de conciencia y de responsabilidad de sus decisiones financieras. 
Figura 3. Promedio de HAH, USF y AEF

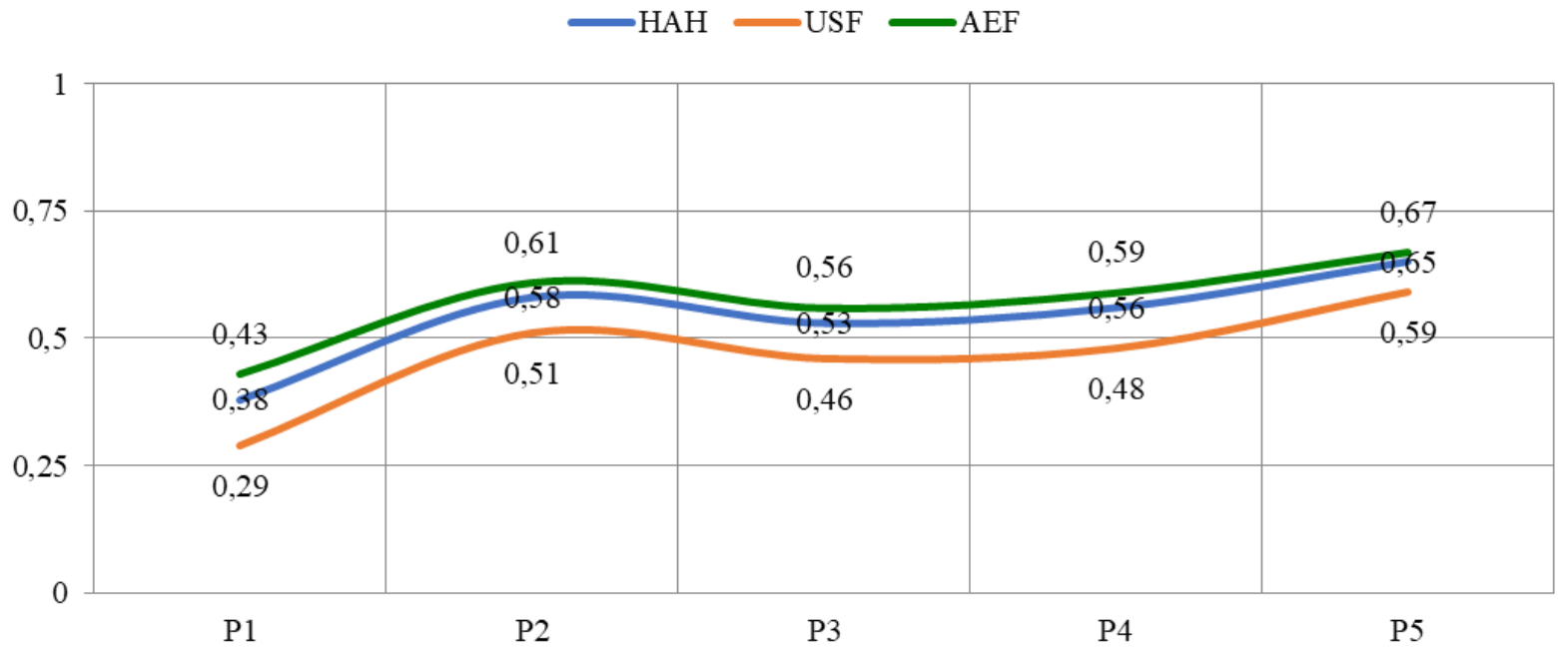

Fuente. Elaboración propia.

El frágil conocimiento financiero que tienen los boyacenses puede influir en el desarrollo de los hábitos financieros que permitan otorgar mayor empoderamiento y mejorar el bienestar económico de estos. Así mismo, esta fragilidad en los conocimientos afecta los progresos en la educación financiera al ralentizar el proceso continuo, la comprensión y el desarrollo de habilidades sobre el funcionamiento de los productos y los servicios financieros. También evitan la generación de cambios de los agentes económicos en su proceder, ya que tener menor información sobre una adecuada gestión de sus recursos financieros afecta negativamente la planificación, el nivel de cultura financiera y la acumulación de riqueza.

Finalmente, se destaca la utilidad del operador FS-OWA, ya que permite obtener ese valor único representativo después de agregar la información según el significado de sus características. Para la investigación permitió tener en cuenta el nivel educativo por cada una de las preguntas, así como asociarlas con vectores de peso que permitan comprender y dar significado sobre el nivel de los conocimientos financieros y cómo pueden ser mejorados teniendo en cuenta el criterio del individuo. 


\section{Discusión}

Con conocimientos financieros sólidos que fortalezcan la educación financiera es posible constituirse en motor de desarrollo económico y de crecimiento de ingresos per cápita en la población. Sin embargo, se aduce de modo generalizado en Latinoamérica un desconocimiento frente a conceptos financieros básicos asociados con tasa de interés, relación riesgo-rentabilidad y comprensión del mercado de capitales (García et al., 2013).

De acuerdo con los resultados obtenidos en la investigación se evidencian estas deficiencias, especialmente en la comprensión de los instrumentos financieros, la capacidad de diversificación y la diferenciación del riesgo. Esta situación se refuerza por el desarrollo de instrumentos financieros complejos, al enfrentar a las personas a decisiones con un mayor grado de responsabilidad y riesgo e incorporar la necesidad de una administración del dinero personal de forma efectiva y en el largo plazo, de modo que desestime el «aprender haciendo» a consecuencia de un impacto adverso en el bienestar económico y social (Lewis y Messy, 2012). Por tanto, los productos financieros vinculan aspectos relacionados con la conveniencia, el poder de compra y una relación de riesgo-rentabilidad (Gautam, 2012).

Con base en lo anterior, las decisiones financieras personales se ven afectadas por los avances de la industria financiera en lo referente a la rápida inclusión de productos y servicios, lo que incrementa el riesgo de las decisiones de gasto mal informadas o contraproducentes en los presupuestos familiares (Bruhn et al., 2013). En este sentido, se evidenció que las dos primeras clasificaciones son consecuentes con la realidad educativa de los boyacenses, si se tiene como ejemplos los depósitos a término y las tarjetas de crédito, las cuales mantienen un mayor reconocimiento en la población. Así, es necesario hacer más sencillo y simple entender los diferentes productos en atención al riesgo-beneficio inherente en ellos.

Por otra parte, las habilidades y la confianza en materia financiera indican consciencia de riesgo, oportunidades financieras (OCDE, 2005) y su diversificación (Lusardi y Mitchell, 2007). En este sentido, los resultados obtenidos denotan dificultad en alcanzar la capacidad de diversificación y la diferenciación del riesgo, lo que conlleva una falta de comprensión 
sobre el riesgo como un elemento determinante frente a las decisiones de financiamiento e inversión.

En un entorno en el que la información se comprenda se podría establecer un escenario de diversificación aplicada, en el cual el inversionista, al poseer información general e instrumentos, hace más fácil una apropiada y orientada toma de decisiones, con lo cual beneficie la profundización y una mejor promoción de los mercados financieros (FonsecaCifuentes y Castaño-Buitrago, 2014).

Si bien existe comprensión frente a la simplicidad de un instrumento bancario, la industria financiera ha desarrollado productos y servicios con un mayor grado de complejidad, lo que posibilita un incremento de la relación riesgo-rentabilidad. Esta complejidad afecta las decisiones de inversión, ya que el consumidor financiero se ve coartado en la toma de decisiones por su desconocimiento y la ausencia de elementos conceptuales básicos que le permitan la diversificación de productos o servicios financieros.

Así mismo, esto conlleva a la adquisición de instrumentos de inversión con una importante aversión al riesgo, mientras que en lo relativo a instrumentos de crédito el efecto contrario - pago de tasas de interés cercanas a la usura dentro del sistema financiero- debido a prácticas financieras deficientes que pueden afectar de forma considerable su bienestar social y financiero, así como el de su núcleo familiar.

Teniendo en cuenta lo anterior, es importante establecer un comparativo sobre los niveles de educación financiera básica con otras regiones del mundo. En este sentido, se toman los resultados del trabajo desarrollado por Lusardi, Michaud y Mitchell (2011) sobre educación financiera (véase la Tabla 13). En esta comparación se destaca que en Boyacá -Colombia, en una puntuación general, solo el 4,3\% de los encuestados respondió las tres preguntas correctas, de modo que fue el tercer país con la más baja puntuación, muy lejos de los mejores países como son Alemania —53,2 \%—, Suiza — 50,1 \%—, Países Bajos y España —44,8 \%— . Así, las puntuaciones sobre los conocimientos relacionados con tipo de interés, inflación y diversificación del riesgo no logran niveles satisfactorios. 
Tabla 13. Educación financiera básica comparativo internacional

\begin{tabular}{|c|c|c|c|c|c|c|c|c|c|c|c|}
\hline \multirow[b]{2}{*}{ Autores } & \multirow[b]{2}{*}{ País } & \multirow[b]{2}{*}{ Años } & \multicolumn{2}{|c|}{ Tipo de interés } & \multicolumn{2}{|c|}{ Inflación } & \multicolumn{2}{|c|}{$\begin{array}{c}\text { Diversificació } \\
\mathrm{n} \text { del riesgo }\end{array}$} & \multirow[b]{2}{*}{$\begin{array}{c}3 \\
\text { correctas }\end{array}$} & \multirow[b]{2}{*}{$\begin{array}{l}\text { al menos } 1 \\
\text { no sabe }\end{array}$} & \multirow[b]{2}{*}{ Observaciones } \\
\hline & & & Correc. & ns & Correc. & ns & Correc. & ns & & & \\
\hline $\begin{array}{l}\text { Romero- Muñoz, } \\
\text { Fonseca-Cifuentes } \\
\text { y Blanco- Mesa } \\
\text { (2021) }\end{array}$ & $\begin{array}{l}\text { Colombia } \\
\text { (Boyacá) }\end{array}$ & 2019 & 37,4 & 36,7 & 43,4 & 30,5 & 16,3 & 38,8 & 4,3 & 52,1 & 1914 \\
\hline Trombetta (2016) & España & 2015 & 85,2 & 8,0 & 70,0 & 20,2 & 56,2 & 37,0 & 44,8 & 22,4 & 500 \\
\hline $\begin{array}{l}\text { Lusardi y Mitchell } \\
\text { (2011) }\end{array}$ & EE. UU. & 2009 & 64,9 & 13,5 & 64,3 & 14,2 & 51,8 & 33,7 & 30,2 & 42,4 & 1488 \\
\hline $\begin{array}{l}\text { Alessie, Van Rooij } \\
\text { y Lusardi (2011) }\end{array}$ & $\begin{array}{l}\text { Países } \\
\text { Bajos }\end{array}$ & 2010 & 84,8 & 8,9 & 76,9 & 13,5 & 51,9 & 33,2 & 44,8 & 37,6 & 1665 \\
\hline $\begin{array}{l}\text { Bucher-Koenen y } \\
\text { Lusardi (2011) }\end{array}$ & Alemania & 2009 & 82,4 & 11,0 & 78,4 & 17,0 & 61,8 & 32,3 & 53,2 & 37,0 & 1059 \\
\hline Sekita (2011) & Japón & 2010 & 70,5 & 12,5 & 58,8 & 28,6 & 39,5 & 56,1 & 27,0 & 61,5 & 5268 \\
\hline $\begin{array}{l}\text { Agnew. Bateman y } \\
\text { Thorp (2013) }\end{array}$ & Australia & 2012 & 83,1 & 6,4 & 69,3 & 13,0 & 54,7 & 37,6 & 42,7 & 41,3 & 1024 \\
\hline $\begin{array}{l}\text { Crossan. Feslier y } \\
\text { Hurnard (2011) }\end{array}$ & $\begin{array}{l}\mathrm{N} . \\
\text { Zelanda }\end{array}$ & 2009 & 86,0 & 4,0 & 81,0 & 5,0 & 27,0 & $2,0^{*}$ & $24,0^{*}$ & 7,0 & 850 \\
\hline $\begin{array}{l}\text { Brown y Graf } \\
\text { (2013) }\end{array}$ & Suiza & 2011 & 79,3 & $2,8^{*}$ & 78,4 & $4,2^{*}$ & $73,5^{*}$ & $\begin{array}{c}13,0 \\
*\end{array}$ & $50,1^{*}$ & $16,9^{*}$ & 1500 \\
\hline $\begin{array}{l}\text { Fornero y } \\
\text { Monticone (2011) }\end{array}$ & Italia & 2007 & $40,0^{*}$ & $28,2^{*}$ & $59,3^{*}$ & $\begin{array}{c}30,7 \\
*\end{array}$ & $52,2^{*}$ & $\begin{array}{c}33,7 \\
*\end{array}$ & $24,9^{*}$ & $44,9^{*}$ & 3992 \\
\hline $\begin{array}{l}\text { Almenberg y } \\
\text { Säve-Söderbergh } \\
(2011)\end{array}$ & Suecia & 2010 & $35,2^{*}$ & $15,6^{*}$ & 59,5 & 16,5 & 68,4 & 18,4 & $21,4^{*}$ & $34,7^{*}$ & 1302 \\
\hline $\begin{array}{l}\text { Arrondel. Debbich } \\
\text { y Savignac (2013) }\end{array}$ & Francia & 2011 & $48,0^{*}$ & $11,5^{*}$ & 61,2 & 21,3 & $66,8^{*}$ & $\begin{array}{c}14,6 \\
*\end{array}$ & $30,9^{*}$ & $33,4^{*}$ & 3616 \\
\hline $\begin{array}{l}\text { Klapper y Panos } \\
\text { (2011) }\end{array}$ & Rusia & 2009 & $36,3^{*}$ & $32,9^{*}$ & $50,8^{*}$ & $\begin{array}{c}26,1 \\
*\end{array}$ & $12,8^{*}$ & $\begin{array}{c}35,4 \\
*\end{array}$ & $3,7^{*}$ & $53,7^{*}$ & 1366 \\
\hline Beckmann & Rumania & 2011 & 41,3 & 34,4 & $31,8^{*}$ & $0,4^{*}$ & 14,7 & 63,5 & $3,8^{*}$ & $75,5^{*}$ & 1030 \\
\hline
\end{tabular}

Correc. $=$ Correcto

Nota.* Indica una formulación ligeramente diferente a las preguntas financieras básicas enumeradas en el texto.

Fuente. Elaboración propia con base en Lusardi y Mitchell, 2014, Trombetta, 2016, y los datos extraídos de la investigación.

Con respecto al concepto de tipo de interés se destaca que en Boyacá el 37,4\% de las personas contestó correctamente la pregunta, siendo la tercera peor puntuación, solo superando a Rusia -36,3\%- y a Suecia -35,2 \%-, en contraste con las puntuaciones obtenidas por Nueva Zelanda -86\%—, España -85,2 \%—, Países Bajos -84,8 \%— y Alemania -82,4 \%-; esto evidencia el incipiente conocimiento sobre el tema de tasas de intereses y su aplicabilidad en operaciones de aritmética básica. 
Para el concepto de inflación se destaca que en Boyacá el 43,4 \% de las personas contestó de forma correcta la pregunta. Aunque es la puntuación más alta de los tres conceptos para la zona, esta es la segunda peor calificación, en general, de modo que solo se supera a Rumanía -31,8 \% - superada con creces Nueva Zelanda - 81 \%-, Alemania -78,4\%-, Países Bajos -76,9\%— y España -70\%—. Con base en esto se puede inferir el limitado conocimiento que se tiene sobre fundamentos económicos.

Para el concepto de diversificación de riesgo se destaca que el 16,3\% de las personas contestó correctamente la pregunta teniendo la puntuación más baja de las tres. Esta puntación es la tercera peor, de modo que solo supera a Rumania -14,7 \%- y a Rusia 12,8 \%-, y se ubica muy lejos de países como Suiza -73,5 \%—, Suecia —68,4 \%—, Francia -66,8 \%- y Alemania -61,8 \%-. Estos resultados muestran aún las deficiencias en el conocimiento del término de diversificación de riesgo y de los productos financieros.

Los resultados presentados revelan las limitaciones que existen sobre la comprensión de conceptos básicos sobre educación financiera en Boyacá -Colombia-. Aunque los datos no contemplan un número significativo de regiones del país, si proporcionan una orientación de lo que ocurre a nivel de conocimiento financiero en regiones pequeñas del país. En este sentido, lo que preocupa es el desconocimiento o no entendimiento de estos términos que se usan indistintamente en la vida cotidiana para la toma de decisiones financieras de las personas.

Finalmente, se evidenció que existe una deficiencia en los procesos de educación financiera de los boyacenses al no comprender los conocimientos básicos para las finanzas personales. Este aspecto denota la urgencia de mejorar este tipo de educación con el fin de tener ciudadanos más conscientes y responsables en sus decisiones financieras. De acuerdo con CIEEF (2017), la educación financiera que se imparta debe ser imparcial, ayudar a entender los beneficios y los riesgos de los diferentes tipos de servicios financieros, de manera que se debe suministrar en un lenguaje claro y sencillo. Así, si se logra una alfabetización oportuna y eficaz, se puede tener a una persona racional y bien informada, que consumirá menos que sus ingresos en épocas de ingresos elevados y de este modo ahorrará para apoyar el consumo 
cuando los ingresos disminuyan (Lusardi y Mitchell, 2014). Por tanto, los conocimientos básicos y especializados contribuyen a una toma de decisiones financieras más informada (Lusardi y Mitchell, 2014).

\section{Conclusiones}

Un aspecto negativo de la falta de conocimiento financiero básico en los boyacenses está relacionado con nivel de conciencia y de responsabilidad de las decisiones financieras y el desarrollo de los hábitos financieros. Según Lusardi y Mitchell (2014), la evidencia empírica muestra que la población aún carece de los conocimientos y las habilidades financieras mínimos requeridos para la toma de decisiones económicas y financieras responsables.

Sin embargo, se observa que la confianza de las personas en sus propias capacidades de toma de decisiones financieras aumenta con la edad mientras que la alfabetización disminuye (Lusardi y Mitchell, 2014), lo cual indica que la educación financiera es esencial en las primeras etapas de la vida y cómo, a medida que se madura, la experiencia y los conocimientos se afianzan para la toma de decisiones responsables. Por tanto, al observar la muestra poblacional, en la que son mayores de edad con uso de servicios financieros, y los niveles de educación se infiere que las estrategias para la alfabetización financiera no obtienen los resultados esperados.

Entre estas decisiones responsables se destaca el hábito de ahorrar, que en los boyacenses están en un acierto del 16,3 \%, y significancias entre los 0,29 y 0,43 puntos, valores que son los más bajos entre todas las preguntas. La motivación del ahorro puede darse para recursos durante el periodo de jubilación, financiar gastos de vivienda y educación, tener una reserva para contingencias y amortizar los recursos con miras a un consumo estable (Melo, Téllez y Zárate, 2006). En este aspecto, apenas se ha investigado cómo se toman las decisiones de ahorro, cómo superan la dificultad de tomar esas decisiones y si tienen los conocimientos financieros suficientes para hacer una elección bien informada (Lusardi y Mitchell, 2008). 
En dichas decisiones de ahorrar influye la distribución del ingreso -influenciado por los estilos de vida - (Figueroa-Delgado, 2009) y eventos inesperados —enfermedad o perdida de trabajo, entre otros- (Miller et al., 2014). Si la persona tiene la posibilidad de ahorrar es importante dónde se salvaguarda ese dinero para mantener el hábito y se tener una reserva futura. Además, la desconfianza por el sistema financiero, en razón a lo complejo o a la baja rentabilidad del portafolio de inversión - ahorro- inclina las preferencias de ahorro a otros servicios tradicionales no ligados a estos.

Por tanto, el hábito del ahorro se ve afectado por diversos factores, de modo que se prioriza, en ocasiones, el consumo de hoy por el consumo futuro. En este sentido, una efectiva educación financiera es necesaria para que las personas interioricen la importancia de empezar a ahorrar desde una temprana edad y asuman de manera informada sus decisiones de ahorro e inversión, tomando en cuenta las opciones que tienen a su alcance y su edad, así como el tipo de pensión o ahorro para la protección a la vejez por el cual se opte (CIEEF, 2017).

Finalmente, para que los boyacenses tomen decisiones financieras más sensatas se requiere que sean capaces de llevar a cabo ciertos cálculos básicos que permitan comparar correctamente las alternativas que se ofrecen (Lusardi y Mitchell, 2008) y comprender la información sobre los productos financieros y el entorno económico que lo rodea.

El interés suscitado en el desarrollo de los conocimientos financieros tiene como propósito promover en la población las competencias y habilidades necesarias para la toma de decisiones de forma acertada y concertada. Dado que el desconocimiento e inexperiencia tiene consecuencias en una inapropiada toma de decisiones y prácticas financieras inconvenientes para los consumidores financieros, esto repercute en el bienestar de la población (García-Bohórquez, 2012). Por tanto, las nociones relacionadas con el conocimiento financiero en pro del mejorar la educación financiera suponen variables fundamentales relacionadas con el conocimiento de conceptos financieros, la capacidad de su entendimiento y las habilidades asociadas a la toma de decisiones. 
De esta manera, en relación con la definición y tomando en consideración las tres variables descritas, una aproximación conceptual sobre su significado permite definirla en los siguientes términos: el conocimiento financiero se entiende como la comprensión de los conceptos financieros, la capacidad y habilidad de entenderlos, para el uso efectivo en la toma de decisiones a corto y largo plazo de su vida.

Por otra parte, la evaluación y la medición permite establecer criterios pertinentes para valorar la percepción en torno al tema, frente lo cual existen metodologías tales como pruebas de porcentaje (Chen y Volpe, 1998; Mandell, 2008; Moore, 2003; Volpe et al., 2002), informes sobre experiencias financieras (Mandell, 2006), pruebas de conocimientos (Agnew y Szykman, 2005), y pruebas de opción múltiple (Lusardi y Tufano, 2009; Lusardi y Mitchell, 2006; 2007; 2008; Van Rooij et al., 2011) entre otros. En el caso de Colombia, Superfinanzas, una metodología desarrollada por la Superintendencia Financiera de Colombia.

De acuerdo con la evidencia metodológica referenciada, se sustraen cinco preguntas que toman como fundamento las metodologías propuestas por Lusardi y Mitchell (2014) y la Superintendencia Financiera de Colombia, en las que se incluyen variables sobre diversificación del riesgo - P1-, inflación - P2-, tasas de interés - P3-, productos e instrumentos financieros $-\mathrm{P} 4-\mathrm{y}$ operatividad de un cheque $-\mathrm{P} 5-$. Estos interrogantes fueron contextualizados de acuerdo con el entorno colombiano y aplicados a una muestra de 1914 personas en trece provincias agrupadas de modo general en cinco de ellas: Sugamuxi -Sugamuxi, Valderrama y Libertad-, Gutiérrez - Gutiérrez, Tundama y Norte-, Centro — Centro y Márquez-, Lengupá -Lengupá, Neira y Oriente- y Occidente -Occidente y Ricaurte-, en Boyacá.

Para esto, los resultados involucran tratamiento descriptivo e híbrido. En el primer caso se asocian conocimientos básicos al entorno regional, el género, la edad y el nivel de educación, mientras que en el segundo se indaga un nivel ideal de respuesta acerca de conocimientos básicos de educación financiera con las variables mediadoras hábito de ahorro, uso de servicios financieros y la mejora de la educación financiera. Se establece en el análisis descriptivo que frente a la operacionalización de un instrumento financiero - cheque- 
existe acierto en un 53,5 \% de las respuestas, mientras que el caso contrario sucede con la diversificación del riesgo con el 16,3 \%. Esto demuestra la necesidad del conocimiento práctico financiero (Hogarth, 2006) que posibilite la toma de decisiones acertada y, por ende, un eficaz cumplimiento del objetivo financiero propuesto, lo cual permitiría la ampliación en la comprensión de las demás variables interrogadas —inflación, tasas de interés y productos financieros-.

Particularizando en el caso regional, la región de Occidente posee los resultados con mayor acierto frente a la diversificación del riesgo, tasas de interés, productos e instrumentos financieros, mientras que, en relación con la operatividad de un cheque. La región de Gutiérrez obtiene un mayor grado de comprensión en el tema. Aunque los resultados no son contundentes - excluyendo la operatividad del cheque- se evidencia que, por áreas geográficas, no existe liderazgo de alguna de estas regiones frente a las variables.

Dentro del estudio, los mayores porcentajes de acierto ocurren cuando el nivel educativo es superior, caso formación posgradual. Esto evidencia una fragilidad en los conocimientos financieros en poblaciones menos educadas. En este sentido, es importante adoptar de forma prioritaria planes de acción que prioricen en segmentos poblacionales, así como mecanismos que coordinen, cooperen y articulen recursos necesarios como política para el cumplimiento de la estrategia de educación financiera nacional (CIEEF, 2017).

En segunda medida, el análisis híbrido caracteriza la información de acuerdo con el grado de importancia para la medición, buscando dar significado a los resultados obtenidos. Se tomaron en consideración los aciertos según el nivel educativo y tres variables de peso: el hábito de ahorro, el uso de servicios financieros y la adecuada educación financiera.

Los resultados muestran que en torno al nivel educativo/adecuada educación financiera (AEF) la mejoría en los niveles de educación financiera conllevaría a superiores habilidades en conocimientos y hábitos en las finanzas personales. Así mismo, la evidencia sobre la relación nivel educativo/hábito de ahorro (HAH) destaca que la mejora en la educación financiera puede ser consecuencia de la apropiada comprensión del ahorro, lo que impactaría positivamente en otros factores tales como el valor del dinero y el riesgo. Finalmente, se 
muestra que el uso de productos y servicios financieros no conlleva el aumento de conocimientos financieros.

Por tanto, estos resultados muestran un deficiente nivel de conocimientos financieros en la población de Boyacá, fundamentado en una menor comprensión de elementos básicos sobre finanzas que tienen efecto en la conciencia y la responsabilidad en la toma de decisiones. Esta situación tiene consecuencias en un menor desarrollo de los hábitos financieros necesarios que permitan mejorar el bienestar personal y el entorno social en general. Igualmente, esto afecta de modo significativo los procesos de organización y planificación financiera, así como elementos relacionados con la cultura financiera.

Este trabajo de investigación tiene algunas limitaciones. Primero, la muestra está restringida a la zona geográfica del departamento de Boyacá, Colombia, por lo cual no se podría relacionar con el comportamiento de la población colombiana. También es importante señalar que los datos recogidos son el reflejo de un momento específico en el tiempo, ya que manifiestan las percepciones respecto a las variables realizadas.

Por consiguiente, en futuras investigaciones se debería incluir otras zonas geográficas regionales y nacionales que permitan hallar diferencias entre estas. Así mismo, realizar varias mediciones en el tiempo, con el fin de observar los cambios en el nivel de conocimiento que puedan tener las personas. Para finalizar, se pueden desarrollar técnicas y metodologías dirigidas a la medición del comportamiento, de las actitudes y las capacidades financieras que permitan conocer y analizar estos elementos y si existe una relación sobre estos.

A pesar de que los avances en inclusión financiera son indudables tanto en oferta de productos como de servicios, los cuales se ajustan de forma heterogénea de acuerdo con las características sociodemográficas de la población, las áreas geográficas, la edad, el género entre otras (Banca de las Oportunidades y Superintendencia Financiera de Colombia, 2018), no son significativos frente a los niveles de conocimiento financiero, con una gran fragilidad en el desarrollo de una educación financiera. Esta fragilidad es evidente en la carencia de conocimientos y habilidades en la población boyacense para la toma de decisiones 
financieras responsables, lo que hace pertinente su fortalecimiento, a través de la educación y la formación con el propósito de generar mayor bienestar de la sociedad (CIEEF, 2017).

Igualmente, se debe considerar que la irrupción de la era digital puede incrementar los obstáculos al acceso y al uso de los servicios financieros, de modo que es necesario la alfabetización financiera digital (Lyons y Kass-Hanna, 2021). Por tanto, se precisa, entre otros cursos de acción, ejecutar la Estrategia Nacional de Educación Económica y Financiera de Colombia (ENEEF), la cual, a través de principios y estándares internacionales, busca la «alianza de educación económica y financiera para los colombianos», y en tal sentido el desarrollo de iniciativas con alianzas estratégicas entre actores públicos y privados que redunden en el afianzamiento de los procesos y los niveles académicos de educación financiera en la población (CIEEF, 2017).

Finalmente, se pretende como futura líneas de investigación realizar un estudio más amplio que incluya más regiones de Colombia y usar otras metodologías que permitan hacer tratamientos de la información subjetiva (Blanco-Mesa, Gil-Lafuente y Merigó, 2018b) que se pueda derivar.

\section{Referencias}

Agnew, J. R.; Szykman, L. R. (2005). Asset allocation and information overload: the influence of information display, asset choice, and investor experience. The Journal of Behavioral Finance, 6(2), 57-70. DOI: https://doi.org/10.1207/s15427579jpfm0602 2

Álvarez-Franco, P. B.; Muñoz-Murillo, A. M.; Restrepo-Tobón, D. A. (2017). Challenges in assessing the effectiveness of financial education programs: the Colombian case. Cuadernos de Administración, 30(54), 155-180. DOI: https://doi.org/10.11144/Javeriana.cao30-54.caef

Baez-Palencia, D.; Olazabal-Lugo, M.; Romero-Muñoz, J. (2019). Toma de decisiones empresariales a través de la media ponderada ordenada. Inquietud Empresarial, 19(2), 11-23. Recuperado de https://bit.ly/3pXueWi

Banca de las Oportunidades; Superintendencia financiera de Colombia. (2018). Reporte de inclusión financiera 2017. Bogotá. Recuperado de https://bit.ly/3m8paxo 
Blanco-Mesa, F.; Gil-Lafuente, A. M.; Merigo, J. M. (2018a). Dynamics of stakeholder relations with multi-person aggregation. Kybernetes, 47(9), 1801-1820. DOI: https://doi.org/10.1108/K$\underline{11-2017-0454}$

Blanco-Mesa, F.; Gil-Lafuente, A. M.; Merigó, J. M. (2018b). New aggregation operators for decisionmaking under uncertainty: an applications in selection of entrepreneurial opportunities. Technological and Economic Development of Economy, 24(2), 335-357. DOI: https://doi.org/10.3846/20294913.2016.1212744

Blanco-Mesa, F.; Gil-Lafuente, A. M.; Merigó, J. M. (2018c). Subjective stakeholder dynamics relationships treatment: a methodological approach using fuzzy decision-making. Computational and Mathematical Organization Theory, 24(4), 441-472. DOI: https://doi.org/10.1007/s10588-018-09284-z

Blanco-Mesa, F.; León-Castro, E.; Acosta-Sandoval, A. (2020). Toma de decisiones estratégicas en entornos inciertos. Revista de Métodos Cuantitativos para la Economía y la Empresa, 30, 79-96. DOI: https://doi.org/10.46661/REVMETODOSCUANTECONEMPRESA.3845

Blanco-Mesa, F.; León-Castro, E.; Gil-Lafuente, A. M. (2019). Aplicaciones matemáticas para la toma de decisiones empresariales en entornos inciertos. Porto: Juruá Editorial. DOI: https://doi.org/10.46661/revmetodoscuanteconempresa.3845

Blanco-Mesa, F.; León-Castro, E.; Merigó, J. M. (2019). A bibliometric analysis of aggregation operators. Applied Soft Computing Journal, 81, 105488. DOI: https://doi.org/10.1016/j.asoc.2019.10548

Braunstein, S. F.; Welch, C. (2002). Financial literacy: an overview of practice, research, and policy. Federal Reserve Bulletin, 88, 445-457. DOI: https://doi.org/10.17016/bulletin.2002.88-11

Bruhn, M.; Leão, L; Legovini, A; Marchetti, R.; Zia, B. (2013). Financial education and behavior formation: large-scale experimental evidence from Brazil (IE 109 No. WPS 6723). Washington.

Chen, H.; Volpe, R. P. (1998). An analysis of personal financial literacy among college students. Financial Services Review, 7(2), 107-128. DOI: https://doi.org/10.1016/S1057$\underline{0810(99) 80006-7}$

Comisión Intersectorial para la Educación y Económica y Financiera. (2017). Estrategia Nacional de Educación Económica y Financiera de Colombia (ENEEF). Bogotá. Recuperado de https://bit.ly/3q2250f

Consejo Nacional de Política Económica y Social. (28 de septiembre de 2020). Documento Conpes 4005. Política nacional de inclusión y educación económica y financiera. Bogotá. Recuperado de https://bit.ly/3pSbt6M 
Cordero, J. M.; Pedraja, F. (2018). La educación financiera en el contexto internacional. Cuadernos Económicos, (95), 239-257. DOI: https://doi.org/10.32796/cice.2018.95.6649

Corporación Andina de Fomento (2015). Encuesta de Medición de Capacidades Financieras en los Países Andinos. Informe para Colombia 2014. Recuperado de https://scioteca.caf.com/handle/123456789/743

DANE. (2007). Censo general 2005. Recuperado de https://bit.ly/3pXurc3

De Beckker, K.; De Witte, K.; Van Campenhout, G. (2020). The role of national culture in financial literacy: cross-country evidence. Journal of Consumer Affairs, 54(3), 912-930. DOI: https://doi.org/10.2139/SSRN.3698656

Departamento Nacional de Planeación. (2011). Plan Nacional de Desarrollo 2010-2014. Prosperidad para todos. Más empleo, menos pobreza y más seguridad. Bogotá. Recuperado de https://bit.ly/3EZ6xDz

Emmons, W. R. (2005). Consumer-finance myths and other obstacles to financial literacy. Saint Louis University Public Law Review, 24(2), 335-362.

Faulcon-Bowen, C. (2002). Financial knowledge of teens and their parents. Journal of Financial Counseling and Planning, 13(2), 93-102.

Figueroa-Delgado, L. 0. (2009). Las finanzas personales. Revista Escuela de Administración de Negocios, (65), 123-144. DOI: https://doi.org/10.21158/01208160.N65.2009.463

Fonseca-Cifuentes, G. P.; Castaño-Buitrago, L. J. (2014). Conocimiento del mercado de valores en las medianas y grandes empresas del departamento de Boyacá: diagnóstico y propuesta estratégica. Inquietud Empresarial, 14(2), 11-29. DOI: https://doi.org/10.19053/01211048.3338

Fox, J.; Bartholomae, S.; Lee, J. (2005). Building the case for financial education. Journal of Consumer Affairs, 39(1), 195-214. DOI: https://doi.org/10.1111/j.1745-6606.2005.00009.x

García, N.; Grifoni, A.; López, J. C.; Mejía, D. M. (2013). La educación financiera en América Latina y el Caribe Situación actual y perspectivas. Caracas: CAF. Recuperado de https://bit.ly/3oWVFjR

García-Bohórquez, N. (2012). El impacto de la educación económica y financiera en los jóvenes: el caso de Finanzas para el Cambio. Borradores de Economía, (687), 1-47. DOI: https://doi.org/10.32468/be.687

Gautam, V. (2012). An empirical study on financial products offered by Indian retail banks to assess the relationship between sale promotions, time to reward receipt, and customer preference. Journal of Relationship Marketing, 11(4), 233-247. DOI: https://doi.org/10.1080/15332667.2012.730969 
Gil-Lafuente, A. M.; Merigó, J. M. (2009). On the use of the OWA operator in the adequacy coefficient. Modelling, Measurement and Control D, 30(1), 1-17.

Goyal, K.; Kumar, S. (2021). Financial literacy: a systematic review and bibliometric analysis. International Journal of Consumer Studies, 45(1), 80-105. DOI: https://doi.org/10.1111/IJCS.12605

Hilgert, M. A.; Hogarth, J. M. (2003). Household financial management: the connection between knowledge and behavior. Federal Reserve Bulletin, 87, 309-322.

Hogarth, J. M. (2006). Financial education and economic development. En Improving Financial Literacy International Conference hosted by the Russian G8 Presidency in Cooperation with the OECD. Moscú, Rusia, 20-30 de noviembre.

Kaiser, T.; Lusardi, A.; Menkhoff, L.; Urban, C. J. (2020). Financial education affects financial knowledge and downstream behaviors ( $\mathrm{N}^{\circ}$ 27057). Cambridge, EE.UU. DOI: https://doi.org/10.3386/W27057

Kim, J.; Kratzer, C. Y.; Leech, I. E. (2001). Impacts of workplace financial education on retirement plans. En J. M. Hogarth (Ed.) Proceedings of the Association for Financial Counseling and Planning Education. (28). Florida: AFCPE.

Kozup, J.; Hogarth, J. M. (2008). Financial literacy, public policy, and consumers' self-protection: more questions, fewer answers. Journal of Consumer Affairs, 42(2), 127-136. DOI: https://doi.org/10.1111/i.1745-6606.2008.00101.x

Levantesi, S.; Zacchia, G.; Levantesi, S.; Zacchia, G. (2021). Machine learning and financial literacy: an exploration of factors influencing financial knowledge in Italy. Journal of Risk and Financial Management, 14(3), 1-21. DOI: https://doi.org/10.3390/jrfm14030120

Lewis, S.; Messy, F. (2012). Financial education, savings and investments: an overview (N WP 22). OECD Publishing. DOI: https://doi.org/10.1787/5k94gxrw760v-en

Lusardi, A. M.; Tufano, P. (2009). Teach workers about the perils of debt. Harvard Business Review, 87(11), 22-24.

Lusardi, A.; Michaud, P.-C.; Mitchell, O. S. (2011). Optimal financial literacy and saving for retirement optimal financial literacy and saving for retirement ( $\mathrm{N}^{\circ}$ WR-905-SSA). DOI: https://doi.org/10.2139/ssrn.1978960

Lusardi, A.; Mitchell, O. S. (2006). Baby boomer retirement security: the roles of planning, financial literacy, and housing wealth. National Bureau of Economic Research Working Paper Series, 54(1), 205-224. DOI: https://dx.doi.org/10.2139/ssrn.1094808 
Lusardi, A.; Mitchell, O. S. (2007). Financial literacy and retirement preparedness: evidence and implications for financial education programs. Business Economics, 42(1), 35-44. DOI: https://doi.org/10.2145/20070104

Lusardi, A.; Mitchell, O. S. (2008). Planning and financial literacy: how do women fare? American Economic Review: Papers \& Proceedings, 98(2), 1-8. DOI: https://doi.org/10.2139/ssrn.1094808

Lusardi, A.; Mitchell, O. S. (2014). The economic importance of financial literacy: theory and evidence. Journal of Economic Literature, 52(1), 5-44. DOI: https://doi.org/10.1257/jel.52.1.5

Lyons, A.; Kass-Hanna, J. (2021). The evolution of financial services in the digital age. En J. Gable; S. Chatterjee (Eds.) Handbook of Personal Finance. (1-18). Berlín: Elsevier. DOI: https://doi.org/10.2139/SSRN.3873370

Mandell, L. (2006). Financial literacy: If It's so important, why isn't It improving? Networks Financial Institute Policy Brief. Nueva York: Elsevier. DOI: https://doi.org/10.2139/ssrn.923557

Mandell, L. (2008). Financial literacy of high school students. En J. J. Xiao (Ed.) Handbook of consumer finance research. (163-183). Nueva York: Springer New York. DOI: https://doi.org/10.1007/978-0-387-75734-6 10

Melo, L.; Téllez, J.; Zárate, H. (2006). El ahorro de los hogares en Colombia. Ensayos Sobre Política Económica, (52), 110-161. DOI: https://doi.org/10.32468/Espe.5203

Miller, M. J.; Reichelstein, J. E.; Salas-Pauliac, C. H.; Zia, B. H. (2014). Can you help someone become financially capable? A meta-analysis of the literature. Washington. DOI: https://doi.org/10.1596/1813-9450-6745

Moore, D. (2003). Survey of financial literacy in Washington State: knowledge, behavior, attitudes, and experiences. Social and Economic Sciences Research Center. Olympia: Washington State Dept. of Financial Institutions.

Olin-Gutiérrez, B. J. (2014). La educación financiera como base para la toma de decisiones personales de inversión (Tesis de maestría). Universidad Autónoma de Querétaro. Santiago de Querétaro, México. Recuperado de https://bit.ly/3GKhEAt

Organización para la Cooperación y el Desarrollo Económicos. (2005). Recomendación sobre los principios y buenas prácticas de educación y concienciación financiera. Recuperado de https://bit.ly/328fDis

Remund, D. L. (2010). Financial literacy explicated: the case for a clearer definition in an increasingly complex economy. Journal of Consumer Affairs, 44(2), 276-295. DOI: https://doi.org/10.1111/i.1745-6606.2010.01169.x 
Romero-Muñoz, J.; Fonseca-Cifuentes, G.; Blanco-Mesa, F. (2021). Analysis and evaluation of financial education in Boyacá. Tunja: Editorial UPTC. DOI: https://doi.org/https://doi.org/10.19053/9789586604833

Rooij, M. van; Lusardi, A.; Alessie, R. (2011). Financial literacy and stock market participation. Journal of Financial Economics, 101(2), 449-472. DOI: https://doi.org/10.1016/i.jfineco.2011.03.006

Schagen, S.; Lines, A. (1996). Financial literacy in adult life: a report to the natwest group. Slough: NFER.

Stone, D. N.; Wier, B.; Bryant, S. M. (2008). Reducing materialism through financial literacy. CPA Journal, 78(2), 12-14. Recuperado de https://bit.ly/3DZ8Rc4

Superintendencia Financiera de Colombia. (2018). Educación al consumidor financiero. Recuperado de https://bit.ly/33BZqDj

Toussaint-Comeau, M.; Rhine, S. L. W. (2000). Delivery of financial literacy programs. Policy Studies, Consumers Issues Research Series. FRB Chicago. Recuperado de https://bit.ly/3dRyOQo

Trombetta, M. (2016). Accounting and finance literacy and self-employment: an exploratory study. Recuperado de https://bit.ly/3dWbj8I

Vanegas, J. G.; Arango-Mesa, M. A.; Gómez-Betancur, L.; Cortés-Cardona, D. (2020). Educación financiera en mujeres: un estudio en el Barrio López de Mesa de Medellín. Revista Facultad de Ciencias Económicas, 28(2), 121-141. DOI: https://doi.org/10.18359/RFCE.4929

Volpe, R. P.; Kotel, J. E.; Chen, H. (2002). A survey of investment literacy among online investors. Financial Counseling and Planning, 13(1), 1-16.

Yager, R. R. (1988). On ordered weighted averaging aggregation operators in multicriteria decisionmaking. IEEE Transactions on Systems, Man, and Cybernetics, 18(1), 183-190. DOI: https://doi.org/10.1109/21.87068

Yager, R. R. (2009). On generalized Bonferroni mean operators for multi-criteria aggregation. International Journal of Approximate Reasoning, 50(8), 1279-1286. DOI: https://doi.org/10.1016/j.ijar.2009.06.004

Zait, A.; Bertea, P. E. (2014). Financial literacy: conceptual definition and proposed approach for a measurement instrument. The Journal of Accounting and Management, 4(3), 37-42. 